\title{
Update of the keratin gene family: evolution, tissue-specific expression patterns, and relevance to clinical disorders
}

\author{
Minh $\mathrm{Ho}^{1+}$, Brian Thompson ${ }^{2+}$, Jeffrey Nicholas Fisk ${ }^{3}$, Daniel W. Nebert ${ }^{4,5}$, Elspeth A. Bruford ${ }^{6,7}$, \\ Vasilis Vasiliou ${ }^{2}$ and Christopher G. Bunick ${ }^{1,8^{*}}$ (D)
}

\begin{abstract}
Intermediate filament (IntFil) genes arose during early metazoan evolution, to provide mechanical support for plasma membranes contacting/interacting with other cells and the extracellular matrix. Keratin genes comprise the largest subset of IntFil genes. Whereas the first keratin gene appeared in sponge, and three genes in arthropods, more rapid increases in keratin genes occurred in lungfish and amphibian genomes, concomitant with land animal-sea animal divergence ( 440 to 410 million years ago). Human, mouse and zebrafish genomes contain 18, 17 and 24 non-keratin IntFil genes, respectively. Human has 27 of 28 type I"acidic" keratin genes clustered at chromosome (Chr) 17q21.2, and all 26 type II "basic" keratin genes clustered at Chr 12q13.13. Mouse has 27 of 28 type I keratin genes clustered on Chr 11, and all 26 type II clustered on Chr 15. Zebrafish has 18 type I keratin genes scattered on five chromosomes, and 3 type II keratin genes on two chromosomes. Types I and II keratin clusters-reflecting evolutionary blooms of keratin genes along one chromosomal segment-are found in all land animal genomes examined, but not fishes; such rapid gene expansions likely reflect sudden requirements for many novel paralogous proteins having divergent functions to enhance species survival following sea-to-land transition. Using data from the Genotype-Tissue Expression (GTEx) project, tissue-specific keratin expression throughout the human body was reconstructed. Clustering of gene expression patterns revealed similarities in tissue-specific expression patterns for previously described "keratin pairs" (i.e., KRT1/KRT10, KRT8/KRT18, KRT5/KRT14, KRT6/KRT16 and KRT6/KRT17 proteins). The ClinVar database currently lists 26 human disease-causing variants within the various domains of keratin proteins.
\end{abstract}

Keywords: Keratin, Intermediate filament, Evolutionary blooms, Gene expression, Gene duplications, Synteny, Markov-chain Monte Carlo (MCMC), MrBayes program to estimate phylogeny

\section{Background}

\section{Intermediate filaments: historical background}

By end of the Cambrian explosion ( 500 million years ago), intermediate filament (IntFil) genes had become well established in the Animalia Kingdom and began expanding rapidly, encoding novel proteins that were

\footnotetext{
*Correspondence: christopher.bunick@yale.edu

${ }^{\dagger}$ Minh Ho and Brian Thompson have contributed equally to this work

1 Department of Dermatology, Yale University, 333 Cedar St., LCI 501, PO Box 208059, New Haven, CT 06520-8059, USA

Full list of author information is available at the end of the article
}

necessary for species survival among metazoans. These IntFil genes played dynamic roles in cell integrity and structural scaffolding-more specifically, to provide mechanical support for plasma membranes where they come into contact with other cells and with the extracellular matrix.

The scientific discovery of IntFils coincided with the birth of structural biology, e.g., William Astbury [1] detected hair and wool diffraction patterns on X-ray photographs in 1931. Building off Linus Pauling's discovery in the 1950s that a protein's secondary structure consists original author(s) and the source, provide a link to the Creative Commons licence, and indicate if changes were made. The images or other third party material in this article are included in the article's Creative Commons licence, unless indicated otherwise in a credit line to the material. If material is not included in the article's Creative Commons licence and your intended use is not permitted by statutory regulation or exceeds the permitted use, you will need to obtain permission directly from the copyright holder. To view a copy of this licence, visit http://creativecommons.org/licenses/by/4.0/. The Creative Commons Public Domain Dedication waiver (http://creativeco mmons.org/publicdomain/zero/1.0/) applies to the data made available in this article, unless otherwise stated in a credit line to the data. 
of $\alpha$-helices and $\beta$-sheets, Francis Crick elucidated that hair keratin's X-ray diffraction patterns were consistent with coiled-coil $\alpha$-helices [2].

IntFils originally were mistaken as part of the "myofibrils group," until Howard Holtzer performed careful electron microscopy experiments and determined that IntFils were $10-\mathrm{nm}$ thick in diameter, as compared with myofibrils (15-nm diameter); hence, the name "intermediate-sized filaments" [3]. In the following years, techniques for isolating and denaturing/reassembling IntFils were fine-tuned for better observation via electron microscopy $[4,5]$. These improved techniques have facilitated a better understanding of IntFil protein structure and the role of IntFils in many human diseases.

By the early 1990s IntFils had been categorized into six classes (i.e., types I, II, III, IV, V \& VI), based on tissuespecific expression patterns, identified by immunofluorescence [6]. Type I "acidic" keratin and type II "basic" keratin expressions are highest in epithelial cells, hair, and nails [7]. Type III IntFil proteins-which include vimentin, desmin, peripherin and glial fibrillary acidic protein-are expressed in mesenchymal, myogenic, neuronal, and glial cells, respectively [8-11]. Expression of type IV neurofilaments is limited to neuronal cells [12]. Type V lamins are expressed in all cells, where they function mostly in the nuclear lamina [13]. Type VI filensin and phakinin were discovered most recently; their expression appears to be limited to the lens of the eye $[14,15]$.

The advent of high-throughput genomic-sequencing technologies has greatly facilitated identification of new IntFil group members [7]. Unfortunately, identification of these new IntFil group members, and in particular the keratin genes, has greatly complicated nomenclature of these genes and has led to substantial confusion. Thus, in 2005, a standardized nomenclature system (https://www. genenames.org/) was established for keratin genes [7]. Due to high similarity in sequence, and vast variations in expression and functionalities among different cell types, functional characterization of some IntFil members continues to be poorly understood.

\section{IntFil proteins: structure and assembly}

The structural domain organization of IntFils is very similar-consisting of a highly conserved $\alpha$-helix central rod domain, flanked by non-helical amino acids at both the $\mathrm{NH}_{2}$-terminus (head) and $\mathrm{COOH}$-terminus (tail) domains. Importantly, the core $\alpha$-helix is constructed in a repeating heptad pattern of amino acids [e.g., $(a b c d e f g)_{n}$ ] with apolar residues existing at positions $a$ and $d$ to ensure a precise coiled-coil dimeric formation between $\alpha$-helices from identical (homodimer) or different (heterodimer) IntFils. The core $\alpha$-helix is divided further into
$1 \mathrm{~A}, 1 \mathrm{~B}, 2 \mathrm{~A}$ and $2 \mathrm{~B}$ sub-domains, which play important roles in coiled-coil formation and higher-order IntFil assembly [16].

Both the homodimeric and heterodimeric coiledcoils form an antiparallel tetramer as the basic building block to form higher-order IntFil assembly units. In order to clarify further interactions between individual IntFil protomers during mature IntFil assembly, Steinert conducted crosslinking nearest-neighbor analyses of keratins - which showed four main modes of tetrameric interactions $[17,18]$; these are termed $\mathrm{A}_{11}(1 \mathrm{~B}-1 \mathrm{~B}$ subdomains in phase), $A_{12}$ (1B-2B subdomains in phase), $A_{22}$ ( $2 \mathrm{~B}-2 \mathrm{~B}$ subdomains in phase), and $\mathrm{A}_{\mathrm{CN}}$ (head-tail interactions) [18].

Herrmann and Aebi proposed three major assembly mechanisms of higher-order IntFil systems based on studies of lamins, vimentin, and keratins [19]. First, the assembly method of lamin was proposed to include longitudinal formation between parallel homodimers in the $\mathrm{A}_{\mathrm{CN}}$ mode-which then enables multiple long strings of lamin to associate laterally through modes $\mathrm{A}_{11}, \mathrm{~A}_{12}$, and $A_{22}$. Second, in contrast, the vimentin method of assembly was proposed that parallel homodimers formed tetramers in antiparallel fashion-using $\mathrm{A}_{11}, \mathrm{~A}_{12}, \mathrm{~A}_{22}$ modes, followed by lateral interaction between tetramers to form the unit length filament (ULF). The ULF comprises 32-mers (i.e., eight tetramers) and is further assembled longitudinally through $\mathrm{A}_{\mathrm{CN}}$ to form a mature vimentin filament. Third, in contrast to vimentin, for keratins both longitudinal and lateral filament assembly apparently happen concomitantly.

These assembly mechanisms were proposed, based on data from negative-stain electron microscopy studies which characterized the in vitro formation of keratins, lamin, and vimentin under physiological conditions [2022]. Stemming from the "divide-and-conquer" ideology from Strelkov, extremely helpful insights into the molecular mechanisms of IntFil assembly were gained by close examination of atomic-resolution crystal structures of lamin and vimentin, and, to a lesser extent, keratins [18, 23]. Recently, the Coulombe, Bunick, and Park groups demonstrated, at the level of atomic resolution, how the $A_{22}$ and $A_{11}$ modes function in keratin, vimentin, and lamin assembly $[16,24,25]$.

Regardless of the proposed mechanism of assembly, it is clear that IntFils form homodimeric or heterodimeric pairs, termed interaction pairs [18]. Similarly, keratin tetramers, the basic building blocks of keratin IntFils, are formed by the antiparallel interaction of two heterodimeric complexes-each comprising one type I and one type II keratin protein (e.g., KRT1/KRT10, KRT5/KRT14, KRT8/KRT18) [5, 26, 27]. One side of the keratin heterodimer has a predominantly hydrophobic character, and 
this forms the major interface between heterodimers in the tetrameric complex [16]; this hydrophobic interface contains a "knob-pocket tetramerization mechanism" on the type II keratin, which is key for driving the $\mathrm{A}_{11}$ tetrameric alignment. This interface between heterodimers is crucial for mature IntFil assembly, as demonstrated by an in vitro study of mutations in type II keratin proteins, which resulted in defective IntFil formation [16].

Given that the IntFil group is quite large, here we limit our discussion primarily to type I and type II keratins. Keratins exhibit unique and interesting evolution, expression patterns, and relevance to human disorders, which we discuss in detail (vide infra). We direct the readers to other informative reviews for a thorough discussion of types III [28], IV [29], V [30] and VI [31] IntFil families.

\section{Main text}

\section{Evolutionary expansion of keratin genes}

Keratins were the first group of IntFils to have their X-ray diffraction pattern discovered [1]. However, from a structural perspective, their molecular functions have been difficult to elucidate; this is in part due to the ability of keratins to form both stable heterodimers and homodimers in vitro-which led to the assumption that this can occur in the living cell (although this has been difficult to confirm) [6].

A phylogenetic tree of the human IntFil group (Fig. 1) reveals that all 18 IntFil genes of types III, IV, V and VI appear to be evolutionarily older than the keratin gene subsets (i.e., IntFil types I \& II). It should be noted that the two synemin protein isoforms in the tree originate from one gene, and the three lamin isoforms are derived from one gene. Note that the IntFil genes of subgroups III, IV, V and VI are scattered among twelve chromosomes (Chr 1, 2, 3, 5, 8, 10,12, 15, 17, 19, 20, 22); this is further evidence that these four IntFil subgroups are evolutionarily very ancient.

The human type II keratin subgroup of 26 genes (Fig. 1) is clustered entirely at Chr 12q13.13, and 27 of the 28 type I keratin genes are clustered at Chr 17q21.2 [32, 33]; the type I KRT18 gene is an exception, located within the type II cluster at Chr 12q13.12. It remains unknown why each of these two clusters have remained together, each on a distinct chromosomal segment. Interestingly, the type I and type II clusters appear to have arisen close to the same evolutionary time. However, the phylogenetic tree suggests that the type I subset might have appeared earlier than the type II subset. This possibility is supported by additional data [vide infra].

A comparable phylogenetic tree in mouse (Fig. 2) shows an evolutionary pattern that is strikingly similar to that in human-except there are 17 IntFil genes (instead of the 18 found in human) in subfamilies III, IV, V and VI that are scattered among thirteen chromosomes (Chr $1,2,3,4,6,7,9,10,11,14,15,18,19)$. In the mouse tree we have included three lamin protein isoforms originating from one gene and three synemin isoforms derived from one gene. The IFFO2 IntFil gene, which is present in human, is absent in mouse; this reflects either a geneduplication event in the human ancestor or a gene-deletion event in the mouse ancestor, after the human-mouse split $\sim 70$ million years ago.

The mouse $B f s p 2$ gene encoding type VI phakanin, located on Chr 9, appears to be associated more closely with the type I cluster in Fig. 2, as was seen with the human phakanin gene (at 3q22.1). The other mouse type VI gene ( $B f_{s p} 1$, encoding filensin) is on Chr 2; the human filensin gene is located at $\mathrm{Chr} 20 \mathrm{p} 12.1$.

With regards to the keratin family, KRT3, KRT37, $K R T 38$, and $K R T 6 C$ are absent from the mouse genome. In contrast, orthologs of KRT42, KRT87, KRT88, KRT90, and KRT222 are present in the mouse genome. The mouse type II keratin subgroup of 26 genes (Fig. 2) is located entirely on Chr 15 , and 27 out of the 28 type I keratin genes are located on Chr 11. As found in human, the one exception in mouse is the type I Krt18 gene, which is located on Chr 15 within the type II cluster; whatever caused this one particular type I gene to be located within the type II cluster in both the human and mouse genomes-while maintaining greater homology with the type I genes-must have taken place before the human-mouse split. All mouse keratin type I and type II genes are syntenic with their human orthologs [https:// www.mun.ca/biology/scarr/MGA2-11-33smc.html]. Examination of keratin genes in all seven additional nonhuman mammals (chimpanzee, macaque, pig, dog, cat,

(See figure on next page.)

Fig. 1 Rooted phylogenetic tree of the human (Homo sapiens) intermediate filaments (IntFils). Protein sequences of the 54 human IntFil types I, II, III, IV, V and VI were retrieved from the Human Intermediate Filament Database and aligned—using maximum likelihood ClustalW Phyml with bootstrap values presented at the node: $>80 \%$, red; 60-79\%, yellow; less than 60\%, black. Branches of the phylogenetic tree are seen at left. The IntFil protein names are listed in the first column. Abbreviations: GFAP, glial fibrillary acidic protein; NEFL, NEFH, and NEFM correspond to neurofilaments L, H \& M respectively; KRT, keratin proteins; IFFO1, IFFO2 correspond to Intermediate filament family orphans 1 \& 2 respectively. The IntFil types are listed in the second column and are color-coded as follows: Type I, grey; Type II, blue; Type III, red; Type IV, gold; Type V, black; Type VI, green, and N/A, non-classified, pink. Chromosomal location of each human IntFil gene is listed in the third column. Known isoforms of synemin and lamin are denoted by the two yellow boxes 


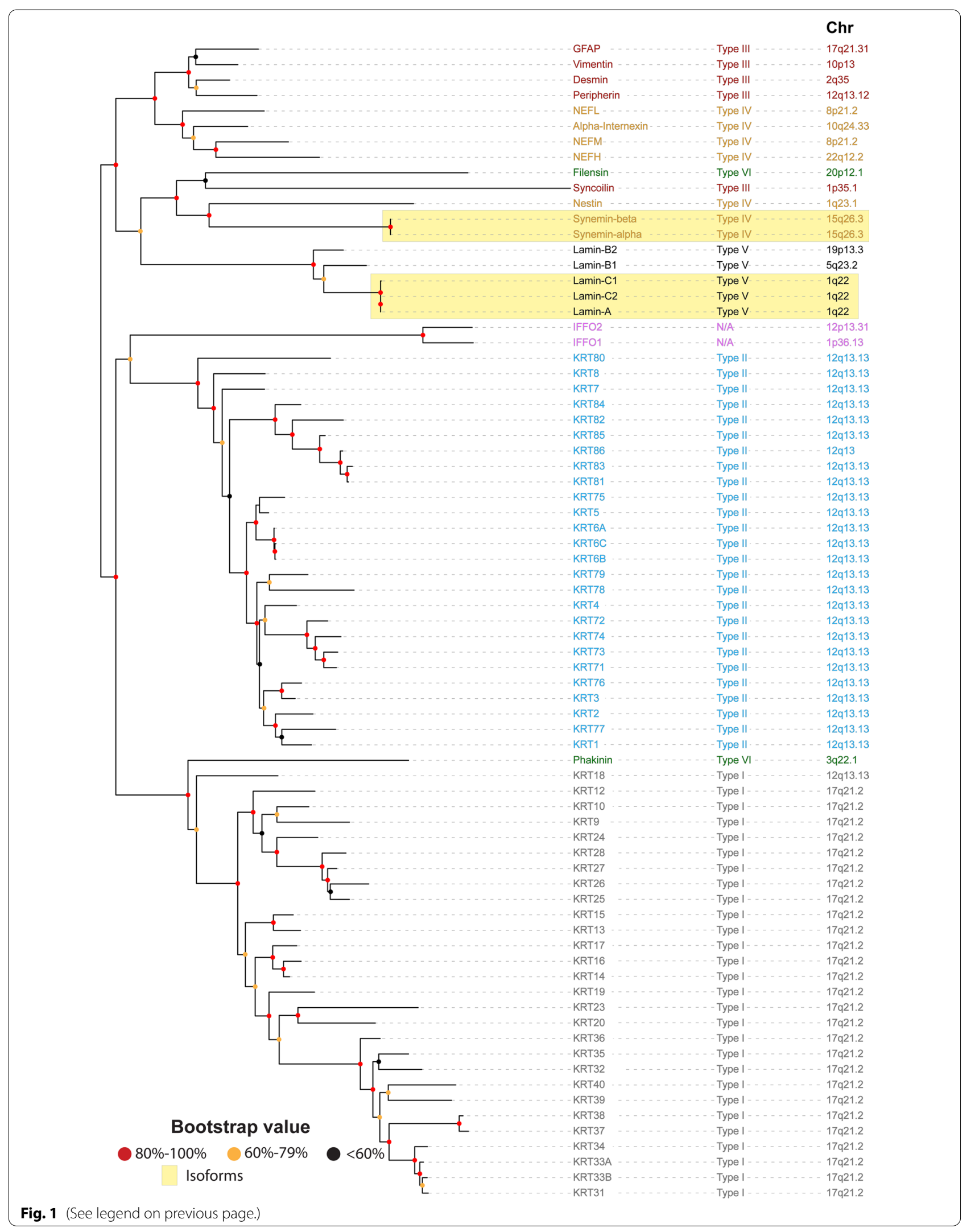


cow, horse) currently registered in the Vertebrate Gene Nomenclature Committee (VGNC, vertebrate.genenames.org) reveals that the two major keratin gene clusters are also conserved in all these species.

\section{Duplications and diversifications of keratin genes}

Paralogs are gene copies created by duplication events within the same species, resulting in new genes with the potential to evolve diverse functions. An expansion of recent paralogs that results in a cluster of similar genesalmost always within a segment of the same chromosome-has been termed 'evolutionary bloom. Examples of evolutionary blooms include: the mouse urinary protein (MUP) gene cluster, seen in mouse and rat but not human [34, 35]; the human secretoglobin (SCGB) [36] gene cluster; and various examples of cytochrome P450 gene (CYP) clusters in vertebrates [37] and invertebrates $[37,38]$.

Are these keratin gene evolutionary blooms seen in the fish genome? Fig. 3 shows a comparable phylogenetic tree for zebrafish. Compared with human IntFil genes (18 non-keratin genes and 54 keratin genes) and mouse IntFil genes (17 non-keratin genes and 54 keratin genes), the zebrafish genome appears to contain 24 non-keratin genes and only 21 keratin genes (seventeen type I, three type II, and one uncharacterized type). Interestingly, the type VI $b f s p 2$ gene (encoding phakinin), which functions in transparency of the lens of the zebrafish eye [39], is more closely associated evolutionarily with keratin genes than with the non-keratin genes; this is also found in human and mouse-which diverged from bony fish $\sim 420$ million years ago. The other type VI IntFil gene in mammals, BFSP1 (encoding filensin) that is also involved in lens transparency [39], appears not to have an ortholog in zebrafish.

Although five keratin genes appear on zebrafish Chr 19, and six keratin genes appear on Chr 11, there is no definitive evidence of an evolutionary bloom here (Fig. 3). If one superimposes zebrafish IntFil proteins on the mouse IntFil proteins in the same phylogenetic tree (Fig. 4), the 24 zebrafish non-keratin proteins show highest homology with the 17 mouse non-keratin proteins; and the 18 zebrafish type I keratin proteins reveal highest homology with the 26 type I keratin proteins in mouse, whereas the three zebrafish type II keratins show highest homology with mouse type II KRT8. These data suggest that both acidic type I and basic type II keratins appeared before the land-sea animal divergence $\sim 420$ million year ago, and both the type I KRT18 and type II KRT8 resemble most closely the ancestral precursor of all other keratins [40].

Furthermore, the basic type II keratin genes might have experienced more selective pressure causing massive gene loss in bony fish, in agreement with a previous report [41], because the type II keratin group in zebrafish has far fewer genes compared with the type I group. Figures 1, 2 and 3 thus suggest that numerous independent gene-duplication events-specifically in the case of the type II keratin cluster of human and mouse keratin genes-occurred evolutionarily before the human-mouse split but after the sea-to-land animal transition.

A gene-duplication event resulting in paralogs is, in and of itself, a selected characteristic, with rates of gene duplication varying across the Tree of Life. Despite being potentially disruptive at both genome and expression levels, the ability of genes to duplicate likely persists as an evolutionarily beneficial device, because it provides species with flexible mechanisms of introducing genetic heterogeneity and allowing members to adapt and thrive during the myriad shifts in environmental pressures experienced by land animals.

From the viewpoint of gene regulation along the linear chromosome, why might evolutionary blooms appear and persist during evolution? One reason for an urgent requirement for many new keratin paralogs-is most likely the critical need for new species of land animals to survive and thrive in the midst of new environmental pressures. There is a second reason. Over a few millions of years, cis-regulatory sequences in noncoding regions (i.e., introns, promoters, enhancers, usually within 10 to $200 \mathrm{~kb}$ of the original regulated gene) might control expression of some, or many, parologous genes located nearby on the same chromosomal segment [42, 43]. In contrast, single gene-duplication events, taking place over much longer periods of evolutionary time, more likely have established their own distinct cis-regulatory noncoding regions - thereby not needing to remain as a cluster at one chromosomal segment; examples would include the type III, IV, V and VI IntFil genes.

\footnotetext{
(See figure on next page.)

Fig. 2 Phylogenetic tree of the inbred C57BL/6J mouse (Mus musculus) IntFil proteins. The same procedures were carried out here as described in the Fig. 1 legend. The IntFil protein names are listed in the first column. Abbreviations: GFAP, glial fibrillary acidic protein; NEFL, NEFH, and NEFM correspond to neurofilaments $\mathrm{L}, \mathrm{H}$ \& M respectively; KRT, keratin proteins; IFFO1 corresponds to IntFil family orphan 1; the evolutionarily most closely related to IFFO is filensin type VI. Chromosomal location of each mouse IntFil gene is listed in the second column. Known isoforms of lamin and synemin are denoted by the two yellow boxes
} 


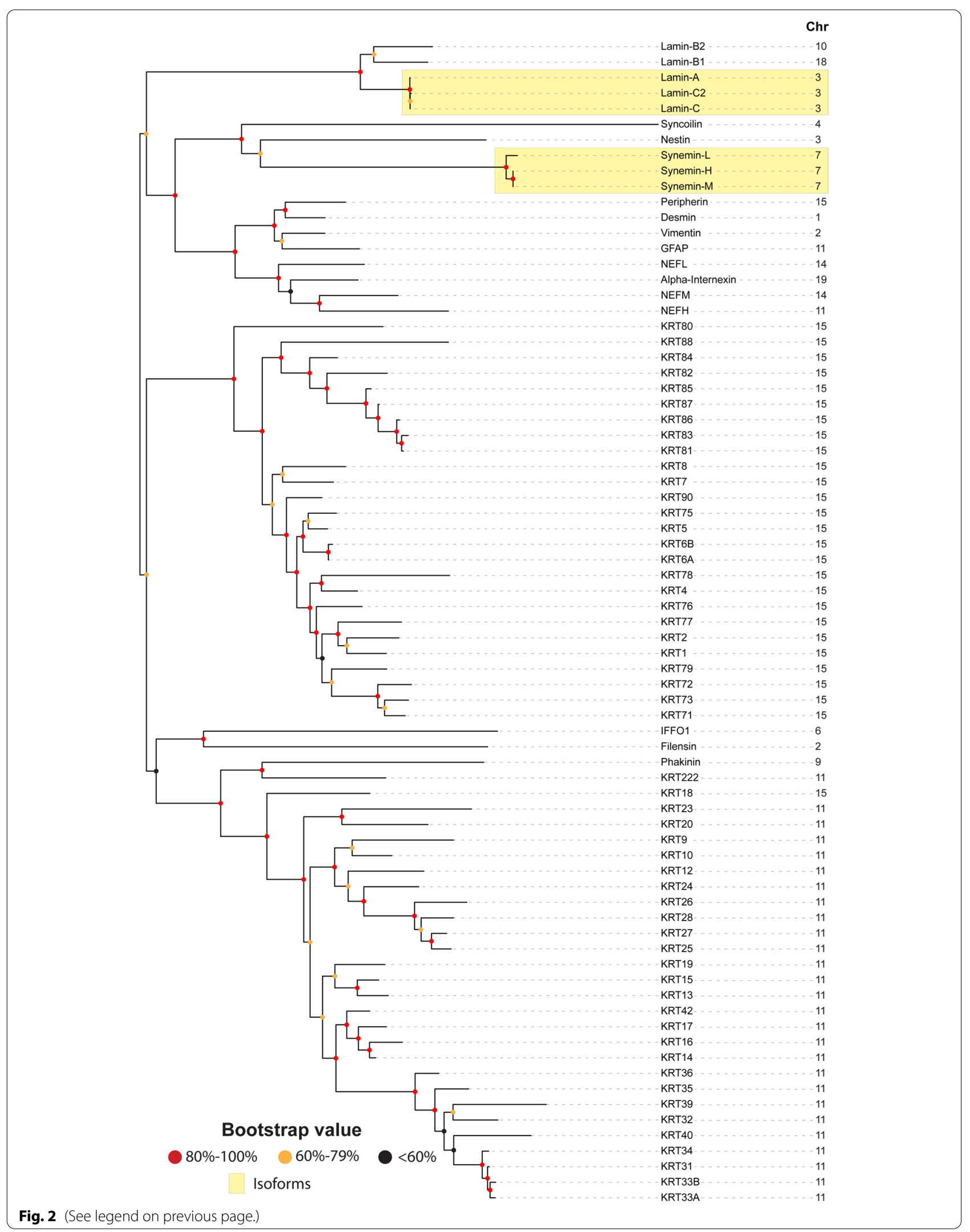




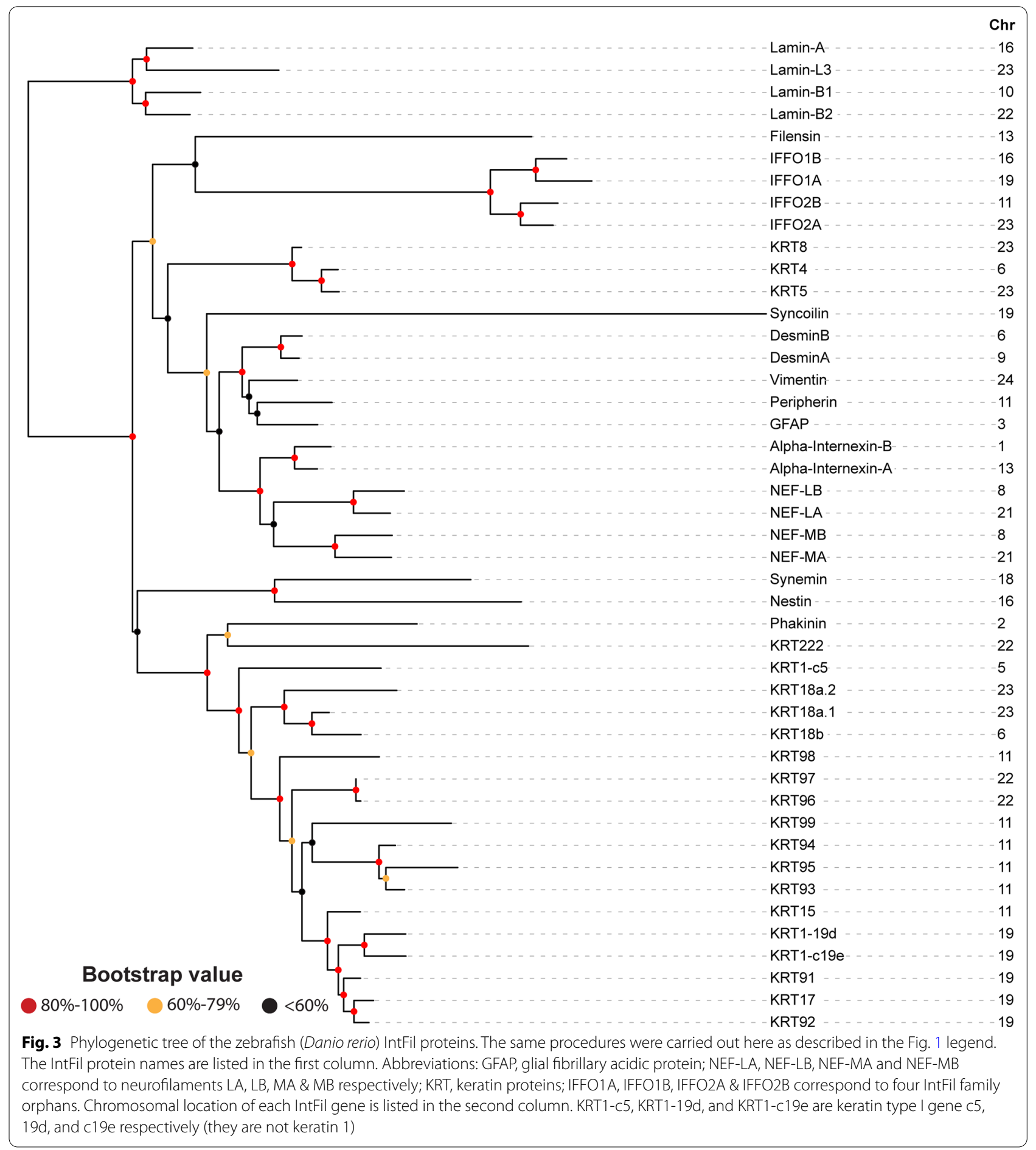

(See figure on next page.)

Fig. 4 Phylogenetic tree of the zebrafish IntFil proteins superimposed on the mouse phylogenetic tree. Names of zebrafish proteins are in red font, mouse proteins in black font. The same procedures were carried out here, as described in the Fig. 1 legend. The IntFil protein names are listed in the first column. "M-" or "Z-" precedes mouse and zebrafish IntFils, respectively. Abbreviations are the same as Figs. 2 and 3. Known isoforms of mouse lamins and synemins are denoted by yellow boxes. The zebrafish's KRT1-c5, KRT1-19d, and KRT1-c19e are keratin type I gene c5, 19d, and c19e respectively (they are not keratin 1) 


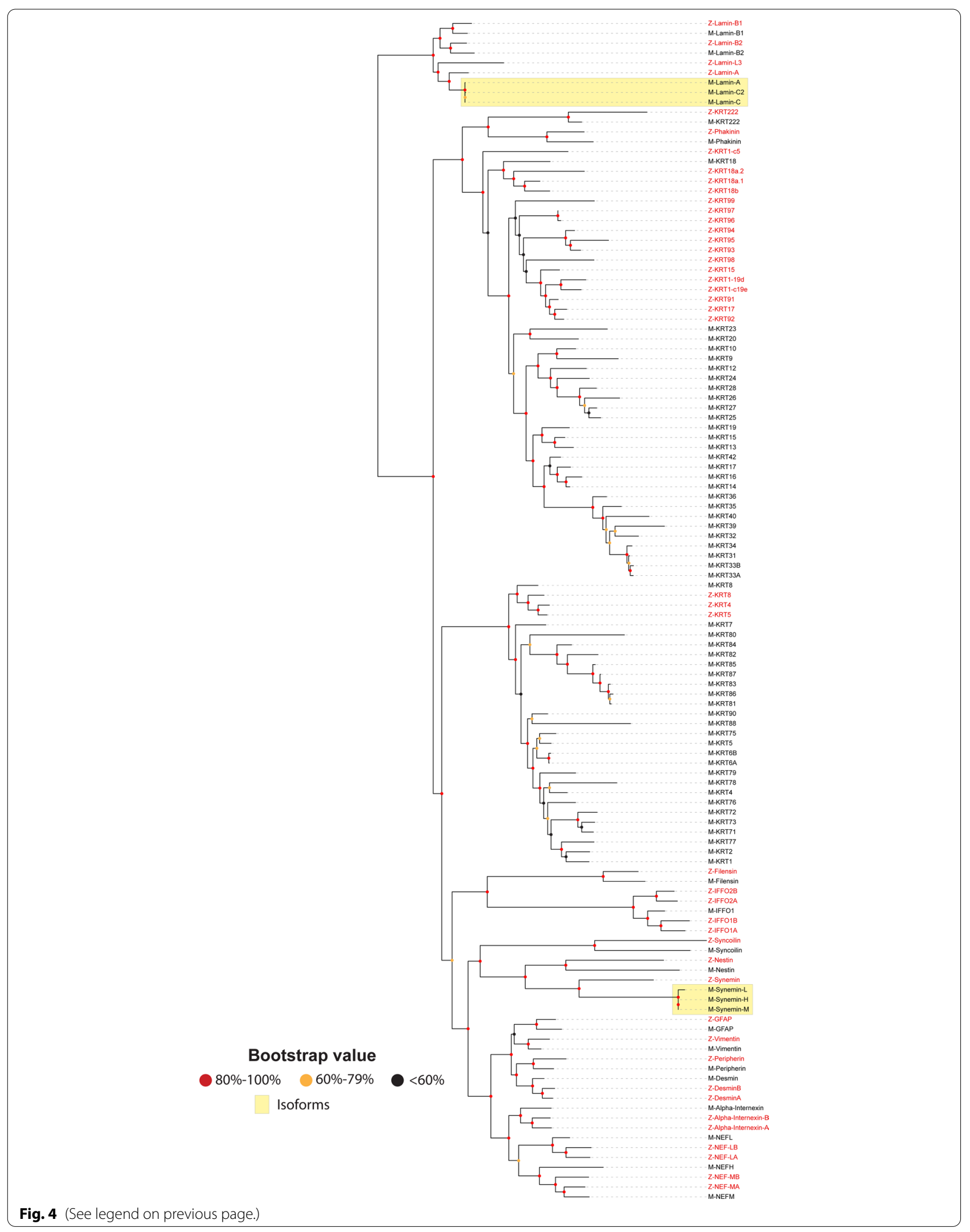




\section{Evolution of keratin gene functions}

Screening 259 species and subspecies in 20 phyla of animals, from jellyfish to human, we examined various features found in type I (Fig. 5a) and type II (Fig. 5b) keratin proteins; we also studied when during the evolutionary history of keratins these features have apparently arisen, disappeared, and, on occasion, reappeared. Between 380 and $\sim 150$ million years (from lungfish to monotremes), dozens of new forms of type I and type II keratin proteins were rapidly co-opted to participate in successfully creating new anatomical structures that were needed in the transition of sea animals to land animals.

The mammalian keratin group members have highly similar rod domains-that are uniquely expressed throughout the epidermis, epithelial cells, and hair follicle. This suggests that small differences among keratin primary sequence are highly specific to a tissue type; this hypothesis is supported by crystallographic data showing that unique amino acids belonging to keratin interaction pairs are primarily positioned along the outer edges of the coiled-coil rod domain, in order to maximize diversity of surface chemistry of the IntFil filament [44].

However, specialized expression, or pairing of IntFil proteins, is not always straightforward. For example, Cetaceans (e.g., whale, dolphin, porpoise) lack expression of KRT24, but, in its absence, the putative partners of KRT24 (i.e., KRT3 and KRT5) interact with KRT14 and KRT12. This finding indicates that keratin proteins can become dispensable in some species, while being repurposed in others $[45,46]$.

We created phylogenetic trees for type I and type II keratin proteins from a broad representation of animal species (Fig. 5). These data suggest that the clade containing the KRT18 (type I) and the KRT80 and KRT8 (type II) proteins is least divergent from the ancient IntFil protein lamin, and most closely resembles precursors for the other members of the keratin group. Localization of the majority of IntFil proteins from earlier Phyla, Classes or Orders (i.e., Cnidaria, Arthropoda, Cephalochordata, Hyperoartia, Chrondrichthyes, Actinopterygii, Coelacanthimorpha and Dipnoi) closest to the ancient protein lamin, and closest to the KRT18, KRT80, and KRT8 clade strengthens the hypothesis that these three keratins were likely the first keratins to form the embryonic epithelium in the Animalia Kingdom [47, 48].

The Fig. 5 trees also suggest that the keratins in species diverging early-relative to human (i.e., Cnidaria and Arthropoda)-have a higher number of proteins related to the ancient IntFil protein, lamin, than to keratins. Within our data, Arthropoda appears to have only one type II keratin (KRT6A) and two type I keratins (KRT13 and KRT14). The type II KRT80 protein in Cnidaria (jellyfish) is apparently lost and then does not reappear until the Testudines Order (turtle). These findings are consistent with the notion that keratin genes can be lost, gained and/or repurposed $[45,46]$.

The type I and type II keratins encoded in the amphioxus (Cephalochordata) genome are also mostly comprised of lamin-like proteins. In contrast, the type I and type II keratins in lamprey (Hyperoartia), cartilaginous fish (Chondrichthyes), and lobe-finned fish with rudimentary legs (Coelacanthimorpha) are closely related to ancestors of type I KRT18 and type II KRT8. Ancestors of the KRT18 and KRT23 type I proteins most likely led to the type I keratins in ray-finned fish (Actinopterygii) and lungfish (Dipnoi). Ray-finned lish and lungfish type II keratins are less divergent from ancestors of the KRT8 proteins.

In the Amphibia Class, type I keratins are closely related to ancestors of 14 keratins (KRT12, KRT17,

Fig. 5 Evolution of animal keratins. Evolutionary relatedness in the type I (a) and II (b) keratin protein sequences from a broad representation of animal species, including human, was reconstructed. The 20 Phyla (or Classes or Orders) that were chosen include: Actinopterygii, ray-finned fishes; Amphibian, frogs-toads-salamanders; Arthropoda, insects-arachnids-millipedes-crusteaceans; Artiodactyla, ungulates (hoofed animals); Aves, birds; Cephalochordata, anphioxus; Cetacea, marine mammals; Chiroptera, bats \& flying foxes; Chondrichthyes, cartilagenous fishes; Cnidaria, jellyfish; Coelacanthimorpha, lobe-finned fishes with rudimenary legs; Crocodylia, crocodiles-alligators; Dipnoi, lungfish; Homo sapiens, modern-day humans; Hyperoartia, lampreys-eels; Marsupialia, kangaroos-wallaby-koalas-oppossums-wombats; Monotremata, platypus-echidna; Rodentia, mice-rats; Squamata, lizards-snakes; and Testudines, turtles, tortoise, terrapins. Protein sequences included in the reconstruction were identified by using the basic local alignment search tool (BLAST) on human keratin proteins against each non-redundant protein database for the clades of interest. For clades more distantly related evolutionarily to humans than Amphibia, only the protein with the highest similarity to human, as determined by the BLOSUM 62 matrix, was included. For Amphibia and clades more closely related to humans than amphibians, the top three proteins with the highest similarity to human —as determined by the BLOSUM 62 matrix - were used for analysis. Evolutionary relationships were inferred using MrBayes under a mixed amino acid model and visualized with the Interactive Tree-of-Life [accessed at itol.embl.de]. The dashed lines link the keratin proteins with their corresponding label. Human keratins are indicated by a red dashed line and red font. Known isoforms are denoted by the yellow boxes. Cnidaria was used as the root for both phylogenetic trees. Labels are written as follows: clades, species, protein name. The "PREDICTED: LOW QUALITY" proteins were labeled with their corrected mutations: yellow lightning bolt indicates insertion/deletion (indel), red lightning bolt indicates nonsense mutation. Clade A is indicated by a pink line. Nodes are colored to indicate posterior probabilities: red, 80-100\%; yellow, 60-79\%; black, $<60 \%$. Details on the animal proteins represented in this phylogenetic tree are contained in Additional file 1: Table S1 and Additional file 2: Table S2 (for type I and type II respectively) 


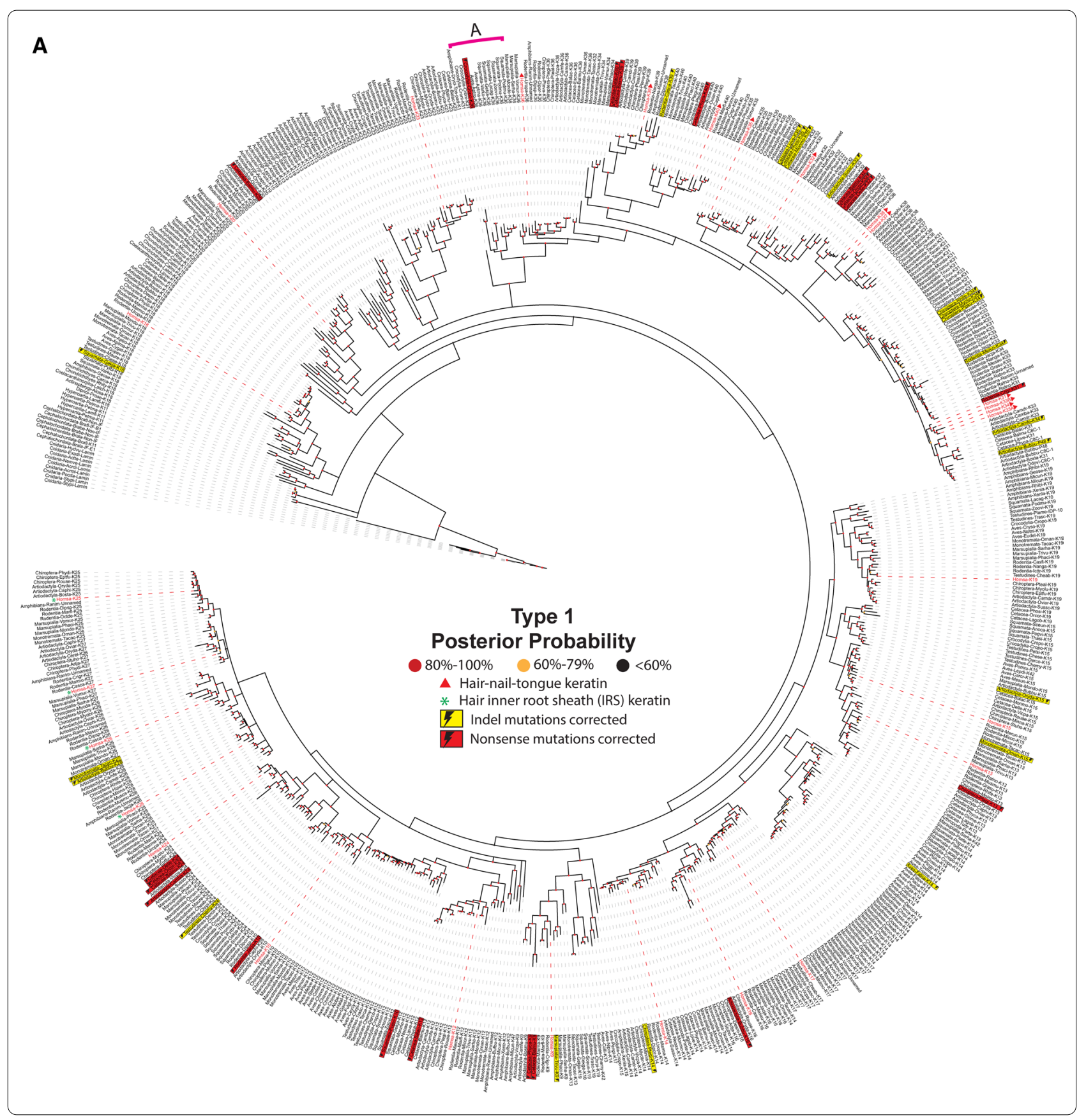




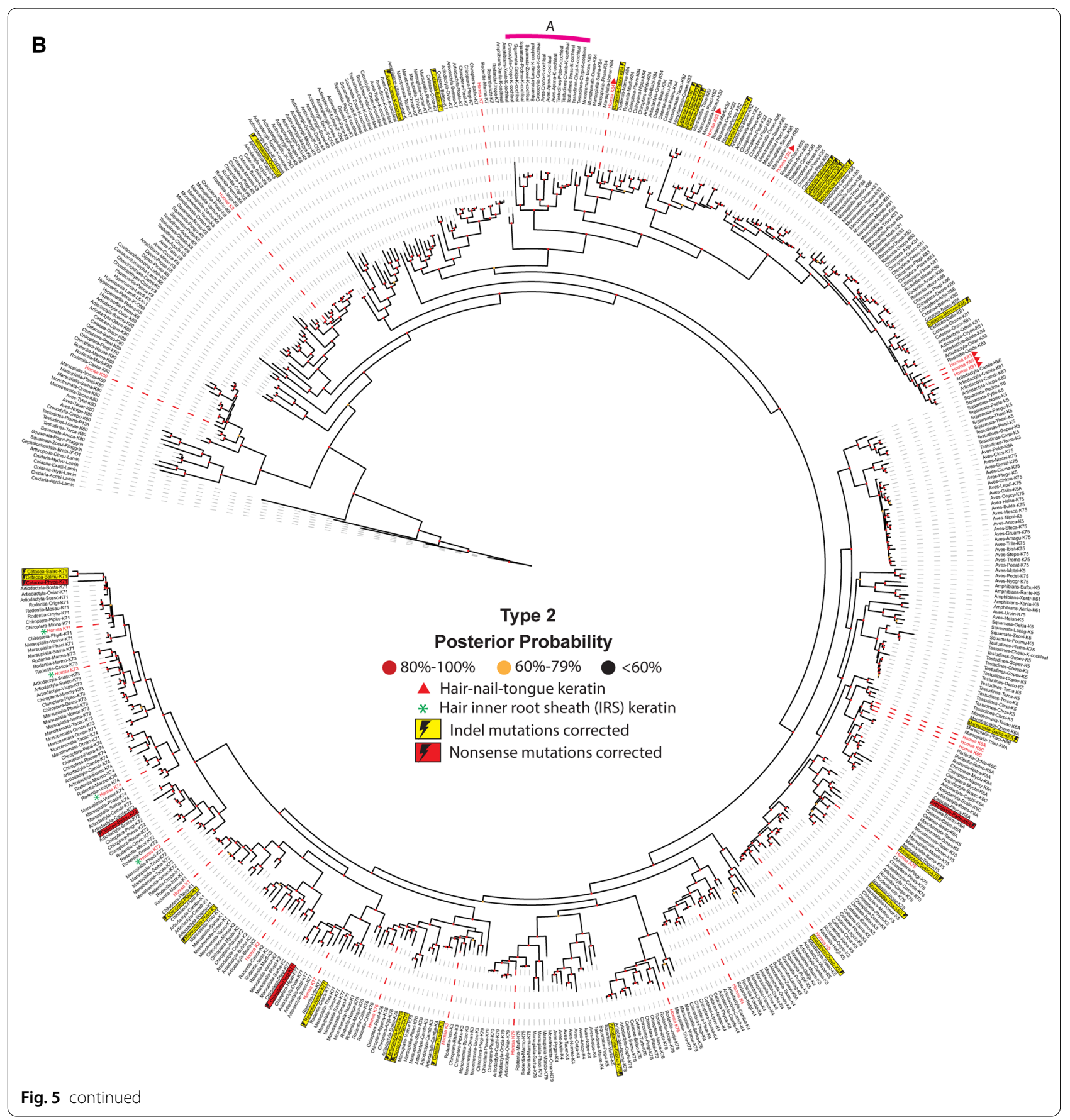

KRT18, KRT19, KRT20, KRT23, KRT25, KRT26, KRT27, KRT28, KRT32, KRT36, KRT39, KRT40), whereas type II keratins are closely related to ancestors of KRT8, KRT7, KRT6A, 6B, and 6C. The type I keratins in Amphibia are strikingly diverse; these observations are consistent with an early split of the phylogenetic tree concordant with the species tree, followed by multiple duplications with subsequent variation and selection. Given that this observation is not replicated in Amphibia type II sequences, it could be posited that type II keratins have broadly experienced more selective pressure, while type I keratins are more robust in structural variation.

The phylogenetic trees also suggest that the earliest hair-nails-tongue (KRT32, KRT36, KRT39, KRT40) and hair inner-root-sheath (IRS) keratins (KRT25, KRT26, KRT27, KRT28) appear to have evolved from the type 
I keratin in Amphibia ancestors (Fig. 5a). The data presented in these phylogenetic trees thus support the previous suggestions that the hair-nails-tongue keratins first appeared in tetrapods (i.e., all vertebrates evolutionarily later than fishes) [49] - to provide protection from friction caused by terrestrial movement and/or to prevent dehydration $[49,50]$. Furthermore, the Fig. 5 trees show that major members of the hair-nails-tongue keratin group (type I: KRT31, KRT32, KRT33A, KRT33B, KRT34, KRT35, KRT36, KRT37, KRT38, KRT39, KRT40; type II: KRT81, KRT82, KRT83, KRT84, KRT85, KRT86) are less divergent from the KRT18, KRT80, and KRT8 ancestral precursors than the group of hair-IRS keratin (type I: KRT25, KRT26, KRT27, KRT28; type II: KRT71, KRT72, KRT73, KRT74); these findings suggest that the hair-nails-tongue, and the hair-IRS, groups appear to have co-evolved, first appearing in the Order Amphibia (Fig. 5a, b). Collectively, these phylogenetic trees support the hypothesis that the massive appearance of ecological function of keratins started in Amphibia, which corresponds to the transition from a water to land lifestyle [50].

Intriguingly, the Fig. 5 data also indicate that the Amphibia ancestral hair-IRS type I keratins (KRT25, KRT26, KRT27, KRT28) and hair-nails-tongue type I keratins (KRT32, KRT36, KRT39, KRT40) disappeared in the Sauropsida clade (Testudines, Crocodylia, Aves, and Squamata) and reappeared again in the Class Mammalia. There are a small number of proteins-from Crocodylia, Aves, Testudines and Squamata-that appear to share the same common ancestor with the mammalian hair-nails-tongue keratins, though they are not directly related (Fig. 5a, b, Clade A). It is likely that this reflects the huge molecular difference between the Sauropsida $\beta$-keratin and the mammalian $\alpha$-keratin and $\beta$-keratin; this also reflects the large differences in skin appendages between Sauropsida (feather, scale, beak and claw) and Mammalia (hair, scale, claw, horn, hoof, and nail) [50].

With regard to marine mammals (i.e., Cetaceans) - the suprabasal plantar-specific keratin genes (type I: KRT10; type II: KRT1, KRT2, KRT77) and sweat gland-specific keratin gene (type I KRT9) are absent or truncated, whereas only basal keratin genes (type I KRT14; type II $K R T 5$,) and hyperproliferation-signal-specific keratin genes (type I KRT17; type II KRT6A,B,C,) are found in the Cetacean genome [51]. This discovery is correlated with the fact that aquatic mammals have thicker basal keratinocyte layers than terrestrial mammals, and that Cetaceans lack the need for footpads and sweat glands (Fig. 5). Note again, that although some keratins are conserved, others have disappeared, reappeared and/ or apparently new ones have arisen-due to the natural selection pressures that facilitate adaptation of new cell type-, tissue- and organ-specific formation; this phenomenon is fundamental in evolution.

Another fascinating example of a missing keratin protein is the absence of the type I keratin KRT24 in whale and walrus - a feature that is thought to play a role in the evolutionary adaptation of these species. Comparative genomics studies have suggested that KRT24 originated in a common ancestor of Amniotes (a clade of tetrapod vertebrates), but then was lost independently in three clades of mammals (i.e., camels, cetaceans, and a subclade of pinnipeds including the eared-seal and walrus) [45, 46]. At first glance, our data (Fig. 5a) would seem to contradict these reports; however, a closer inspection of the Cetacean KRT24 gene sequence revealed that it contains multiple premature stop codons. These would likely result in either elimination of the messenger RNA by nonsense-mediated decay, or production of a nonfunctional protein that would rapidly undergo proteasomal degradation. The existence of these premature stop codons in the sequence of KRT24 in Cetaceans supports the notion that KRT24 is dispensable; this discovery also may provide a mechanism by which keratins 'disappear' from the genome (i.e., slow accumulation of mutations) [52]. Furthermore, from our phylogenetic tree, we have found the possible existence of truncated KRT32, KRT39 and KRT40 proteins in the Cetacean group; these findings suggest further the mutational inactivation of these keratins among the members of the Infraorder Cetacea.

In conclusion, the appearance-disappearance-reappearance of keratin features-throughout evolutionary history-support the notion that the gain-of-function and loss-of-function of certain types of keratins (Fig. 5) are likely to be involved in evolutionary adaptation [45]. If the same rigorous examination across the Animalia Kingdom - as was done here for the keratin clusters (Fig. 5) - were to be carried out for the MUP [34, 35], SCGB [36], and CYP [37, 38] evolutionary blooms, perhaps similar patterns of gain-of-function and loss-offunction (as a function of evolutionary time) might also become apparent. Consistent with the observations of a higher tendency of truncated keratins appearing in the type I keratins, the rates of evolution of new keratin proteins, specifically type I, coincide with the rates of evolution of all metazoans, and, ultimately, mammals.

\section{Tissue-specific expression of human keratins Tissue-specific expression patterns of keratin pairs}

Using data retrieved from the Genotype-Tissue Expression (GTEx) project [53], we reconstructed the expression of keratins throughout the human body in a tissue-specific manner (Fig. 6). Interestingly, the majority of keratin genes (i.e., KRT3, KRT6C, KRT9, KRT12, KRT20, KRT24, KRT25, KRT26, KRT27, KRT28, KRT31, 


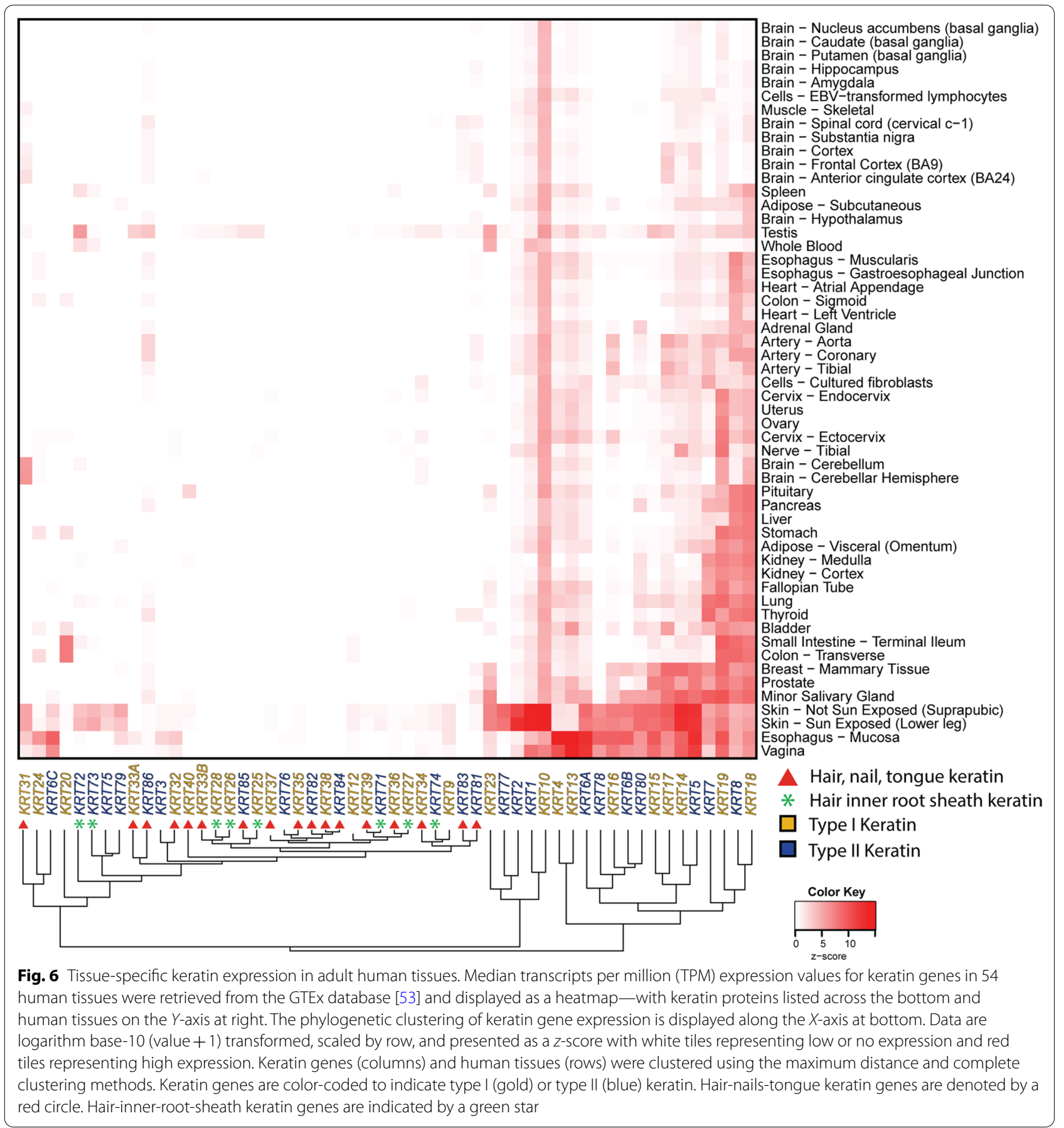

KRT32, KRT33A, KRT33B, KRT34, KRT35, KRT36, KRT37, KRT38, KRT39, KRT40, KRT71, KRT72, KRT73, KRT74, KRT75, KRT76, KRT79, KRT81, KRT82, KRT83, KRT84, KRT85, KRT86) - lack highly substantive expression in the majority of human tissues listed in GTEx.

It is important to note that the GTEx database does not contain keratin expression data on hair, nails and tongue, which are known to be tissues with exceptionally high expression of many keratins. In fact, all keratin genes that lack marked expression in any human tissue in GTEx are those with notable expression in either hair, nails, or tongue (Fig. 6). It is likely that, if GTEx had data on these other tissues, one would see high expression for these tissues.

As anticipated, clustering of gene expression patterns revealed similarities in the tissue-specific expression patterns of the five keratin-interaction pairs (i.e., KRT1/ 
KRT10, KRT8/KRT18, KRT5/KRT14, KRT6/KRT16 and $K R T 6 / K R T 17$ genes). However, tissue-specific expression patterns of KRT6A, KRT6B and KRT6C were only moderately similar to that of $K R T 17$ (vide infra). Given the importance of keratin-interaction pairs for their function, below we provide detailed discussions solely of the expression patterns for those genes involved in these five keratin pairs.

\section{KRT1/KRT10}

Both $K R T 1$ and $K R T 10$ display expansive expression patterns with expression in every tissue within the GTEx database (Fig. 6). This diverse expression pattern is likely due to their roles in differentiated epithelial cells [54]. However, despite their functions as a pair, the tissue-specific expression levels of KRT1 and KRT10 are only weakly positively correlated $(\rho=0.54, P=2.70 \mathrm{e}-05)$. Even with their weak correlation, tissue-specific expression patterns between KRT1 and KRT10 did cluster next to one another-indicating that their expression patterns were more similar to each other than to any other keratin.

$K R T 1$ expression is lower than KRT10 expression in every tissue, except for whole blood [transcripts-permillion (TPM) of 16.1 vs 10.5]. As shown in Fig. 6, KRT10 is the most highly expressed keratin gene in subcutaneous adipose tissue, arteries (aorta and tibial), all brain regions except for cerebellum and cerebellar hemispheres, cell cultures [cultured fibroblasts and Epstein-Barr virus (EBV)-transformed lymphocytes], sigmoid colon, atrial appendage and left ventricle of heart, skeletal muscle, and skin (both sun-exposed of lower leg and non-sun-exposed of suprapubic region).

The observation of KRT10 expression in every tissue within the GTEx database is in agreement with numerous prior reports of expression in skin [55], breast [56], testis [57], cervix [58], thymus [59] and vagina [60]; and with the finding that expression of a transgene driven by the KRT10 promoter was observed in stomach, small intestine, cecum, colon, spleen, and pancreas [61]. While KRT1 expression is well established in skin integrity [55, 62], colonic mucosa [63], kidney [64] and vagina [65], the GTEx data indicate that KRT1 has a much more expansive expression pattern than is suggested by the literature. These expression data also raise the question as to whether KRT10 is expressed in terminally-differentiated epithelial cells [66].

\section{KRT8/KRT18}

Both KRT8 and KRT18 are expressed in every tissue within the GTEx database (Fig. 6). This diverse expression pattern is likely due to their role in simple epithelial cells [54, 67]. In contrast to KRT1/KRT10, KRT8 and KRT18 tissue-specific expression levels were very strongly positively correlated ( $\rho=0.89, P=5.5 \mathrm{e}-19)$, and clustered next to each other. KRT8 was the most highly expressed keratin in esophagus, both in the gastroesophageal junction and the muscularis. KRT8 expression is greater than any other keratin in three specific locations: the gastroesophageal junction of esophagus, atrial appendage of heart, and left ventricle of heart.

Similarly, KRT18 was the most highly expressed keratin gene in several tissues: adipose tissue (visceral omentum), adrenal gland, coronary artery, renal cortex and medulla, liver, pancreas, pituitary, spleen, and thyroid. Thus, as expected, $K R T 18$ expression is higher than $K R T 8$ in every tissue except for the aorta, bladder, esophagus (gastroesophageal junction), atrial appendage of the heart, transverse colon, and terminal ileum of small intestine.

KRT8 expression in the GTEx database is in agreement with previous reports that described expression in uterus, vagina, bladder [60], pancreas, liver [68], fetal heart tissues [69], mammary tissue [70], colon, small intestine, esophagus, kidney, lung [71], ovary [72], stomach, thyroid and, prostate [73]. KRT18 expression patterns in GTEx are in agreement with previous reports in bladder [54], mammary tissue [70], intestine [54, 74], pancreas [74], liver [54, 74, 75], lung [67, 75], esophagus [76], colon [54, 75, 77], kidney, cervix, spleen, brain and skin [75].

\section{KRT5/KRT14}

Both KRT5 and KRT14 are expressed in most tissues within the GTEx database (Fig. 6). Again, this is consistent with their known expression in stratified and simple epithelium [74]. Tissue-specific expression levels of KRT5 and KRT14 are strongly positively correlated $(\rho=0.81$, $P=2.2 \mathrm{e}-13)$ and clustered next to one another. Similarities in their tissue-specific expression levels and patterns are expected, given their role as interaction partners in heterodimeric pairs. Neither of these keratin genes is the most highly expressed keratin in any of the tissues listed within the GTEx database. KRT5 expression is higher than KRT14 expression in most tissues-except for subcutaneous adipose, aorta, coronary and tibial arteries, the caudate region of brain, the spinal cord (cervical C-1), breast/ mammary, minor salivary gland, skeletal muscle, tibial nerve, terminal ileum of small intestine, skin (sun-exposed and non-sun-exposed), and cultured fibroblast cells.

The KRT5 expression pattern described in the GTEx database is in agreement with previous reports of KRT5 expression in differentiating adipose-derived stem cells [78], whole blood [79], esophagus, skin, bladder, mammary tissue $[54,80]$, cervix [81], lung [80, 82], prostate, liver, pancreas, stomach and salivary gland $[80,83]$. The finding that KRT14 expression occurs in every tissue, 
except for the renal medulla, is in agreement with previous reports demonstrating KRT14 expression in uterus, vagina, bladder [60] esophagus [54], mammary tissue, lung, prostate and salivary gland [54, 80]. Furthermore, failure to find KRT14 expression in renal medulla is consistent with a previous report [80].

\section{KRT6/KRT16}

As expected, tissue-specific expression levels were strongly correlated with the keratin-interaction pairings KRT6 (KRT6A, KRT6B and KRT6C) and KRT16 (Fig. 6): KRT6A/KRT16 $(\rho=0.83, P=1.1 \mathrm{e}-14) ; K R T 6 B / K R T 16$ $(\rho=0.83, P=1.5 \mathrm{e}-14)$; and KRT6C/KRT16 $(\rho=0.80$, $P=3.6 \mathrm{e}-13)$. It should be noted, however, that the correlation between $K R T 6 B$ and KRT16 is heavily influenced by the large number of genes having low or no expression, which will be similarly classified near the bottom of the ranked-order list.

GTEx data indicate that $K R T 6 A$ is expressed in every tissue. In contrast, $K R T 6 B$ is not expressed in the brain region except in cerebellum, nor is it in EBV-transformed lymphocytes, the left ventricle of heart, renal cortex and medulla, skeletal muscle, or whole blood. In addition to the same tissues that lack KRT6B expression, KRT6C is not expressed in subcutaneous or visceral (omentum) adipose, adrenal gland, cultured fibroblasts, endocervix, sigmoid and transverse colon, gastroesophageal junction of the esophagus, atrial appendage and left ventricle of heart, or the liver, lung, tibial nerve, pancreas, stomach, and thyroid.

KRT16 is expressed in every tissue except for renal medulla, and the following brain regions: hypothalamus, frontal cortex, anterior cingulate cortex, hippocampus, caudate, nucleus accumbens, putamen, substantia nigra, and amygdala (Fig. 6). Interestingly, there were only eight tissues with higher expression of KRT16 than any of the three KRT6 keratins: the adipose subcutaneous, aorta, coronary and tibial regions of the artery, breast mammary tissue, cervix endocervix, tibial nerve, and prostate (Fig. 6).

The finding that KRT6 genes (KRT6A, KRT6B or $K R T 6 C)$ are expressed in every tissue is in agreement with previous reports of KRT6 expression in uterus, vagina, bladder $[60,80]$, skin $[54,84]$, esophagus, liver, lung, pancreas, prostate, salivary gland, and stomach $[54,80]$. That KRT16 expression is found in most tissues is consistent with previous reports of expression in cervix [85], esophagus [54], and skin [86]. However, the expansive KRT16 expression pattern in human tissues in GTEx is in disagreement with previous reports that failed to find KRT16 expression in hepatocytes, colon, small intestine, mammary gland ducts [54], bladder [54,
87], and prostate [88]. Interestingly, the expression pattern of $K R T 16$ is shown to be more closely related to that of $K R T 6 A$ and $K R T 6 B$ than to the expression pattern of KRT17.

\section{KRT6/KRT17}

Given that KRT6 and KRT17 are an interaction pair, it was unexpected to see $K R T 17$ expressed in every tissue, whereas only KRT6A (and not KRT6B or KRT6C) is similarly expressed in every tissue (Fig. 6); however, their tissue-specific expression levels were only weakly positively correlated $(\rho=0.59, P=2.6 e-6)$. Despite the high number of tissues having undetectable $K R T 6 B$ and $K R T 6 C$ expression, both genes exhibited weakly positive correlations in tissue-specific expression patterns with $K R T 17(K R T 6 B=\rho=0.61, P=6.8 \mathrm{e}-7 ; K R T 6 C=\rho=058$, $P=5.1 \mathrm{e}-6)$. Interestingly, the strengths of these correlations are almost identical to those of KRT6A and KRT17; this is likely due to the fact that tissues having low or no expression will similarly be ranked consistently near the bottom. This would result in correspondingly weak positive correlations.

However, when comparing tissue-specific expression patterns between KRT17 and KRT6A, KRT6B or KRT6C by analyzing their clustering patterns, it became apparent that KRT17 and KRT6A are more similar than KRT17 and either KRT6B or KRT6C. KRT17 expression is higher than $K R T 6 B$ or $K R T 6 C$ expression in every tissue within the GTEx database, except for the muscosal esophagus and vagina. KRT17 expression is higher than KRT6A expression in every tissue in the GTEx database-except for subcutaneous and visceral (omentum) adipose, the cerebellum and nucleus accumbens (basal ganglia) of brain, ectocervix, transverse colon, gastroesophageal junction, mucosa and muscularis of the esophagus, Fallopian tube, atrial appendage and left ventricle of heart, liver, skeletal muscle, ovary, pancreas, terminal ileum of the small intestine, spleen, stomach, uterus, and vagina.

The discovery that KRT17 is expressed in every tissue in GTEx is in agreement with previous reports of KRT17 expression in skin, esophagus, mammary gland [54], bladder, prostate [89], lung [90], and ovary [91]. However, the expansive $K R T 17$ expression that we found in the GTEx database is different from previous reports that failed to detect KRT17 expression in colon, small intestine, liver, salivary gland, esophagus, stomach, intestine [54, 89], cervix [92], and thyroid [93].

\section{Possible reasons for discrepancies}

The data that we have collected from GTEx disagree with some of the findings from previous publications. The main reason is undoubtedly due to advances in imaging 
Table 1 Distribution of 26 disease-causing variations in human keratin protein domains

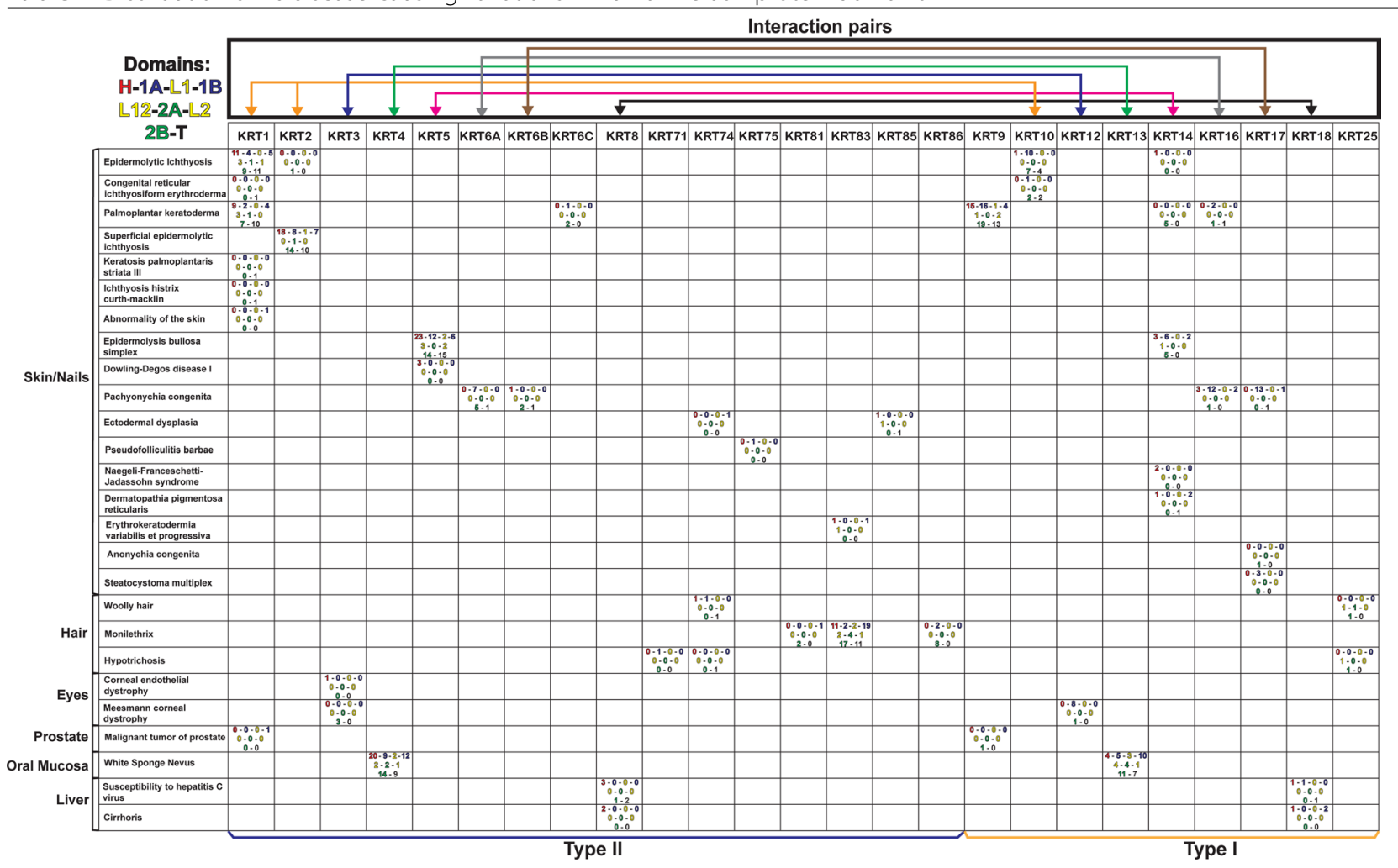

Searching the ClinVar database for coding variations in 54 human type I and type II keratin genes revealed 26 variations classified as pathogenic (this includes susceptibility to hepatitis $\mathrm{C}$ virus). Names of the disorders caused by variations in keratin-coding sequences are shown in the left column. Keratin genes are listed in the row at top. Domain locations for pathogenic variants are designated as: Top row: Head (red); 1A (blue), L1 (gold), 1B (blue); Middle row: L12 (gold), 2A (green), L2 (gold); Bottom row: 2B (green), Tail (black). Keratin-interaction partners are indicated by colored lines as follows: KRT1, KRT2/KRT10 (orange), KRT3/KRT12 (blue), KRT4/KRT13 (green), KRT5/KRT14 (pink), KRT6A/KRT16 (grey), KRT6B/KRT17 (brown), KRT8/KRT18 (black). The number of variants in a keratin domain, associated with a given disorder, is displayed. Type II keratin proteins are shown at left and are indicated by a blue line along the bottom of the figure. Type I keratin proteins are exhibited at right and denoted by a gold line along the bottom of the figure

and scientific methodology. Indeed, most of the previous findings were derived from immunostaining to detect the signal of protein expression only and failed to detect: KRT10 in bladder [94] or uterus epithelial cells [60]; KRT8 in cervix, spleen, or testis [58, 73]; KRT16 in colon, small intestine, mammary gland duct [54], bladder [54, 87], or prostate [88]; KRT17 in colon, small intestine, liver, salivary gland, esophagus, stomach, intestine [54, 89], cervix [92], or thyroid [93].

Moreover, some older studies used 2D-electrophoresis, which is much less sensitive and can give false-negative signals; for example, neither KRT5 nor KRT14 expression was detected in brain, muscle, ovary, pancreas, spleen, or testis [80]. Furthermore, lack of knowledge about highly similar proteins meant gene-specific probes were not used; for example, in the case of KRT6, this could limit the ability to correctly distinguish the expression of $K R T 6 A, K R T 6 B$, and KRT6C [95].

It is also possible that a false-positive signal might arise from the use of anti-keratin antibodies that have similar epitopes, e.g., using anti-KRT5/KRT6 to detect KRT5 or KRT6 [95]. Recently, Marc's group identified possible cross-contamination occurring for the GTEx data during library preparation [96]. This certainly raises concerns regarding validity of some data presented in the GTEx database, and it will be important-as newer large-scale datasets emerge-to cross-validate the keratin gene expression findings described herein from GTEx.

\section{Involvement of keratin proteins in human disease}

In the ClinVar database, analysis of human diseasecausing variations within keratin proteins reveals that for diseases of the skin-such as epidermolytic ichthyosis, superficial epidermolytic ichthyosis, epidermolysis bullosa simplex, palmoplantar keratoderma, and whitesponge nevus-more changes occur in the head $(\mathrm{H})$, tail $(\mathrm{T})$, and rod domains $(1 \mathrm{~A}, 1 \mathrm{~B}, 2 \mathrm{~A}, 2 \mathrm{~B})$ of type II keratins (KRT1, KRT2, KRT5, KRT6) than of type I keratins (Table 1). However, the disease-causing variants observed in type I keratins (KRT9, KRT10, KRT13, KRT14, KRT16, KRT17) occur mostly in the rod domains (1A, 1B and 
2B), except in the case of palmoplantar keratoderma. These data suggest that explanations of the phenotype (i.e., the human disorder) caused by each of these mutations might range from disrupting the dimer, tetramer, and higher-order formation, to the IntFil-interacting interface with its dimeric partner.

As indicated in Fig. 6, a large number of keratins are expressed in hair-nails-tongue. Therefore, it is expected that mutations in these keratin genes would lead to diseases of these tissues. Indeed, this is the case: defects in hair-nails-tongue keratin proteins-such as KRT25, KRT71, KRT74, KRT81, KRT83, KRT86-are involved in diseases of the hair and nails (Table 1). More specifically, variants in the KRT74 and KRT85 genes are associated with ectodermal dysplasia; KRT25 and KRT74 variants are associated with woolly hair; KRT75 variants are associated with pseudo-folliculitis barbae; KRT81, KRT83 and $K R T 86$ variants are associated with monilethrix; KRT17 variants are associated with anonychia congenita; and variations in KRT25, KRT71 and KRT74 are associated with hypotrichosis (Table 1).

The GTEx database indicates that many keratin genes, including KRT1, KRT10, KRT8, KRT18, KRT6A, KRT17, KRT5 and KRT14, are expressed in every tissue (Fig. 6). Interestingly, variants in keratin genes do not appear to cause disease in the vast majority of these tissues, aside from KRT8/KRT18 (Table 1). KRT8/KRT18 variations listed in the ClinVar database may be involved in nonalcoholic steatohepatitis (NASH), in oxidative stress to the liver, indirectly leading to cirrhosis [97, 98], and in increased formation of fibrosis during chronic hepatitis $\mathrm{C}$ infection [99].

These data, nevertheless, beg the question as to why alterations of ubiquitously expressed keratin genes (such as KRT8/KRT18) cause disease only in liver, and not in a multitude of other tissues. One possibility is that such changes disturb interactions between keratins and binding partner(s) (i.e., keratin-associated proteins)-rather than disrupting the integrity of the KRT8/KRT18 protein dimer itself; this hypothesis would make most sense if those mutated amino acids are located at the solventexposed molecular surface, or if the IntFil surface chemistry is altered.

Interactions of KRT6 with KRT16 or KRT17 are very intriguing. The evolutionary change of KRT1/KRT10 to KRT6/KRT17 in aquatic mammals (Cetaceans) suggests that KRT6/KRT17 might be associated with life in cold water [51], in which a thickened basal layer of epidermis would be beneficial (Fig. 5). In early studies on ridged skin of the human palm, KRT17 was found to be expressed in the basal layer of the primary epidermal ridge, whereas KRT16 expression occurs across the secondary epidermal ridge; this finding indicates that KRT17 plays a larger role than KRT16 in maintaining a high proliferation signal under high-stress conditions [86]. Accordingly, Coulombe and colleagues discovered that KRT17 has a high capacity to induce hyperproliferation signals-through the STAT3 and $14-3-3 \sigma$ pathways [100, 101]. In contrast, KRT16 function appears to be more connected with KRT6 and maintenance of cellular mitochondrial organization [102], as well as with innate immunity [103].

Variations in the KRT6A, KRT6B, KRT16 and KRT17 genes are, in large part, associated with a rare disease related to thickening and abnormal shaped of fingernail and toenail, pachyonychia congenita (PC) (Table 1); while an absence of the Krt16 gene in mouse causes thickening skin of palms and feet, palmoplantar keratoderma (PPK) [104]. PC-related variants are more frequently associated with type I genes KRT16 (18 variants) and KRT17 (15 variants) than with the type II genes KRT6A (13 variants), KRT6B (4 variants), and $K R T 6 C$ (novariants). Furthermore, PC- and PPK-related variations primarily result in perturbation of the $1 \mathrm{~A}$ and $2 \mathrm{~B}$ domains of the keratin proteins, suggesting they distort either filament formation or how these keratins interact with other intracellular proteins [105].

Recent evidence suggests that KRT8 overexpression on the cell surface might enhance cell adhesion to the extracellular matrix-raising questions about involvement of KRT8 in cancer-cell-signaling pathways [106]. These studies suggest that IntFils may be potential targets for future therapeutics in prevention of viral infection and cancer treatment. Non-keratin IntFils have also recently been implicated in many diseases-including COVID19 infection and cancer-cell signaling. For example, the IntFil type III vimentin was found to be upregulated in human cells infected with SARS-CoV and is suspected to facilitate entry of the virus into host cells [107]. Furthermore, a recent article deposited in bioRxiv suggests that extracellular vimentin acts as a critical component of the SARS-CoV-2 spike protein-ACE2 complex and that antibodies against vimentin can prevent SARS-CoV-2 infections in vitro [108].

\section{Conclusions}

Intermediate filaments (IntFils), and in particular keratins, have been a focus of researchers for well over 50 years. IntFils are critical in intracellular and extracellular support to create distinct cell-types, tissues, organs, appendages, and body shapes. Our understanding of these multi-functional cytoskeleton proteins has advanced dramatically with the development of new investigative technologies. With respect to posttranslational keratin filament assembly, we now know that discrete molecular interactions can regulate higher-order keratin structures (e.g., a knob-pocket tetramerization mechanism in the 1B domain of type II keratins). 
Paralogs (genes created by duplication events which often lead to diverse functions) - that have expanded rapidly in evolutionary time such that they exist as a cluster within a segment of the same chromosome-have been termed 'evolutionary blooms.' By examining human, mouse, and zebrafish phylogenetic trees, we show that keratin type I and type II clusters exist in genomes of human and mouse but not fish. These conserved clusters have also been found in seven other mammals (chimpanzee, macaque, pig, dog, cat, cow, horse) currently registered in the Vertebrate Gene Nomenclature Committee (vertebrate.genenames.org). Screening 259 species and subspecies in 20 phyla of animals, from jellyfish to human, we identified keratin proteins that appear to have arisen, disappeared, and sometimes reappeared. Between $\sim 380$ and $\sim 150$ million years, dozens of new forms of type I and type II keratin proteins were rapidly recruited in creating new anatomical structures needed during the transition of sea animals to land animals.

Analysis of keratin evolution also suggests that the type II keratins experienced more selective pressure than the type I keratins throughout time and thus type II keratins likely played a greater role in speciation of the animal kingdom. Despite experiencing less selective pressure than type II keratins, type I keratins nonetheless were involved in diversification of species and sub-speciation. Ultimately, the evolution of keratins reflects the evolutionary history of the animal kingdom.

Despite having similar coiled-coil structural folds, keratin proteins exhibit distinct surface chemistries that enable unique, diverse roles for keratins in extraand intra-cellular functions-critical during embryonic development and establishing basic human physiology (e.g., epidermal skin barrier integrity). This functional diversity is directly correlated with multiple human diseases that can occur when humans acquire new variants/ mutations in keratin genes, resulting in defective assembly, or altered keratin protein function. It is apparent that IntFils are involved in the etiology and/or progression of rare skin diseases, cancer, and possibly even COVID-19.

Interestingly though, the range of diseases caused by mutations in keratins is narrower than would be expected-given the expansive expression patterns of keratins in all cell-types of the human body. This peculiarity suggests that redundancies may exist among keratins, and perhaps among other IntFils, that remain to be elucidated. It is anticipated that studies which leverage next-generation technologies [e.g., CRISPR/Cas9, artificial intelligence (AI), machine learning (ML), and deep learning (DL)] to investigate these mysteries will have enormous therapeutic potential by uncovering novel mechanisms by which keratins might be targeted.

\section{Methods}

\section{Maximum likelihood phylogenetic inference}

Sequences were aligned in MAFFT using the L-INSI local pair methodology with 10,000 iterative alignment steps. Evolutionary models were determined using ModelFinder as implemented in IQTree, using Bayesian Information Criteria (BIC) to select the optimal model and gamma rate categories. Maximum Likelihood Phylogenetic trees were then constructed using the optimal model in IQTree; 10,000 Ultrafast Bootstrap permutations were performed to measure tree consistency. Due to the potential for model violations, each bootstrap tree was further optimized using a hill-climbing nearest neighbor interchange (NNI) protocol. Ultrafast Bootstrap Scores more closely resemble probabilistic measures than standard non-parametric bootstraps-but still should not be interpreted as strict probabilities of branching support.

\section{Bayesian inference of animal keratin phylogenies}

Multiple sequence alignments were generated using the interactive Fast-Fourier Transform method in MAFFT, building the guide tree five times in the progressive stage with 10,000 refinement iteration cycles. Evolutionary relationships were estimated by Markov-chain Monte Carlo (MCMC) using MrBayes and an aminoacid-rate matrix averaged across 10 canonical distributional models. Each phylogenetic tree was inferred by two independent MCMC simulations lasting for $2.0 \times 10^{7}$ iterations, sampling every 1000 generations in parallel using the BEAGLE library. Sufficient sampling of the posterior distributions of each parameter was evaluated-using effective sample size (ESS) values, with ESS values $>100$ indicating adequate sampling of target parameters. Parallel-chain convergence was checked, using the within-chain and between-chain variance potential scale reduction factor (PSRF). Independent runs were assessed for convergence, and appropriate levels of burn-in visually, through visual inspection of the marginal posterior probabilities versus the generation state. The sampled posteriors from the two independent executions were then combined to generate a maximum clade-credibility tree-summarizing the posterior distribution of estimated evolutionary relationships and branch lengths.

\section{Tissue-specific expression}

Median tissue-specific expression values for human keratin genes were retrieved from the GenotypeTissue Expression (GTEx) database v8 [53] for all available human tissues. Only keratin genes with 
transcripts-per-million (TPM) counts of $\geq 0.1$ were counted as "significantly expressed" in that tissue, whereas genes that failed to meet this criterion were classified as "not expressed" in that tissue. TPM counts were loaded into the Galaxy web platform [109], and the heatmap2 program within this platform was used to create heatmaps with the following options "-transform logarithm base 10 (value +1 ), -scale by row, -cluster columns maximum distance and complete."

\section{Abbreviations}

BFSP1: Filensin; BFSP2: Phakinin; CYPs: Cytochrome P450 monooxygenases; GTEx: Genotype-Tissue Expression project; IFFO1 \& 2: Intermediate filament family orphans 1 \& 2; IntFil: Intermediate filament; KRT: Keratin; MCMC: Markov-chain Monte Carlo; MUPs: Mouse urinary proteins; PC: Pachyonychia congenita; PPK: Palmoplantar keratoderma; SCGBs: Human secretoglobins; TPM: Transcripts-per-million; ULF: Unit length filament.

\section{Supplementary Information}

The online version contains supplementary material available at https://doi. org/10.1186/s40246-021-00374-9.

Additional file 1. Table containing genes included in the phylogenetic tree for Type I Keratins.

Additional file 2. Table containing genes included in the phylogenetic tree for Type II Keratins.

\section{Acknowledgements}

We thank Dr. Zeng Wang from the Department of Biostatistics (Yale School of Public Health) for useful discussions.

\section{Authors' contributions}

$\mathrm{W}$ and $\mathrm{CB}$ conceived the project. MH and BT performed investigative studies, method development, data retrieval and analyses. JF performed phylogenic analyses. MH and BT wrote the initial manuscript draft with assistance from JF. $\mathrm{MH}$ produced figures. DN, EB, W and CB analyzed the final data and wrote the manuscript with $\mathrm{MH}, \mathrm{BT}$ and JF. All authors have read and approved the final manuscript.

\section{Funding}

This work was supported by the US National Institutes of Health (NIH)/ National Institute of Arthritis and Musculoskeletal and Skin Diseases (NIAMS) under Award Numbers K08 AR070290, R03 AR076484, and R01 AR079428 (to CGB), EY017963 (to WV), TL1 TR001864 (to BT), EY022313 (to BT), U24 HG003345 (to EAB), and P30 ES006096 (to DWN). It was also supported by the Wellcome Trust UK 208349/Z/17/Z (to EAB). The content is solely the responsibility of the authors and does not necessarily represent the official views of the National Institutes of Health.

\section{Availability of data and materials}

All data used in this publication are publicly available at GTEx database v8 (https://gtexportal.org/home/), Zebrafish Information Network database (https://zfin.org/), Human Intermediate Filament database (http://www.inter fil.org/), Universal Protein Knowledgebase (https://www.uniprot.org/), and Mouse Genome Informatics database (http://www.informatics.jax.org/).

\section{Declarations}

Ethics approval and consent to participate Not Applicable.

\section{Consent for publication}

Not Applicable.

\section{Competing interests}

The authors declare that they have no competing interests.

\section{Author details}

${ }^{1}$ Department of Dermatology, Yale University, 333 Cedar St., LCI 501, PO Box 208059, New Haven, CT 06520-8059, USA. ² Department of Environmental Health Sciences, Yale School of Public Health, New Haven, CT 06511, USA.

${ }^{3}$ Program of Computational Biology and Bioinformatics, Yale University, New Haven, CT 06511, USA. ${ }^{4}$ Departments of Pediatrics and Molecular and Developmental Biology, Cincinnati Children's Research Center, Cincinnati, OH 45229, USA. ${ }^{5}$ Department of Environmental Health and Center for Environmental Genetics, University of Cincinnati College of Medicine, Cincinnati, $\mathrm{OH} 45267$, USA. ${ }^{6} \mathrm{HUGO}$ Gene Nomenclature Committee (HGNC), EMBL-EBI, Wellcome Genome Campus, Hinxton, Cambridge CB10 1SD, UK. ${ }^{7}$ Department of Haematology, University of Cambridge, Cambridge CB2 OXY, UK. ${ }^{8}$ Department of Molecular Biophysics and Biochemistry, Yale University, New Haven, CT 06520, USA.

Received: 5 November 2021 Accepted: 17 December 2021

Published online: 06 January 2022

\section{References}

1. Street WTAA. X-ray studies of the structure of hair, wool, and related fibres. I. General Philos Trans R Soc Lond A. 1931;230:75-101.

2. Crick FH. Is alpha-keratin a coiled coil? Nature. 1952;170(4334):882-3.

3. Ishikawa H, Bischoff R, Holtzer H. Mitosis and intermediate-sized filaments in developing skeletal muscle. J Cell Biol. 1968;38(3):538-55.

4. Steinert PM, Gullino MI. Bovine epidermal keratin filament assembly in vitro. Biochem Biophys Res Commun. 1976;70(1):221-7.

5. Aebi U, et al. The fibrillar substructure of keratin filaments unraveled. J Cell Biol. 1983;97(4):1131-43.

6. Steinert PM, Roop DR. Molecular and cellular biology of intermediate filaments. Annu Rev Biochem. 1988:57:593-625.

7. Schweizer J, et al. New consensus nomenclature for mammalian keratins. J Cell Biol. 2006;174(2):169-74.

8. Franke WW, et al. Different intermediate-sized filaments distinguished by immunofluorescence microscopy. Proc Natl Acad Sci USA. 1978:75(10):5034-8.

9. Lazarides E, Hubbard BD. Immunological characterization of the subunit of the 100 A filaments from muscle cells. Proc Natl Acad Sci USA. 1976;73(12):4344-8.

10. Portier MM, de Nechaud B, Gros F. Peripherin, a new member of the intermediate filament protein family. Dev Neurosci. 1983;6(6):335-44.

11. Bignami $A$, et al. Localization of the glial fibrillary acidic protein in astrocytes by immunofluorescence. Brain Res. 1972;43(2):429-35.

12. Hoffman PN, Lasek RJ. The slow component of axonal transport Identification of major structural polypeptides of the axon and their generality among mammalian neurons. J Cell Biol. 1975;66(2):351-66

13. Aebi $U$, et al. The nuclear lamina is a meshwork of intermediate-type filaments. Nature. 1986;323(6088):560-4.

14. Merdes A, et al. Filensin: a new vimentin-binding, polymerizationcompetent, and membrane-associated protein of the lens fiber cell. J Cell Biol. 1991;115(2):397-410.

15. Merdes A, Gounari F, Georgatos SD. The 47-kD lens-specific protein phakinin is a tailless intermediate filament protein and an assembly partner of filensin. J Cell Biol. 1993;123(6 Pt 1):1507-16.

16. Eldirany SA, Ho M, Hinbest AJ, Lomakin IB, Bunick CG. Human keratin 1/10-1B tetramer structures reveal a knobpocket mechanism in intermediate filament assembly. EMBO J. 2019;38(11):e100741. https://doi. org/10.15252/embj.2018100741.

17. Steinert PM, et al. Keratin intermediate filament structure. Crosslinking studies yield quantitative information on molecular dimensions and mechanism of assembly. J Mol Biol. 1993;230(2):436-52.

18. Eldirany $S A$, et al. Recent insight into intermediate filament structure. Curr Opin Cell Biol. 2021;68:132-43.

19. Herrmann $\mathrm{H}$, Aebi U. Intermediate filaments: molecular structure, assembly mechanism, and integration into functionally distinct intracellular Scaffolds. Annu Rev Biochem. 2004;73:749-89. 
20. Herrmann H, et al. Structure and assembly properties of the intermediate filament protein vimentin: the role of its head, rod and tail domains. J Mol Biol. 1996;264(5):933-53.

21. Karabinos A, et al. The single nuclear lamin of Caenorhabditis elegans forms in vitro stable intermediate filaments and paracrystals with a reduced axial periodicity. J Mol Biol. 2003;325(2):241-7.

22. Heitlinger $\mathrm{E}$, et al. Expression of chicken lamin B2 in Escherichia coli: characterization of its structure, assembly, and molecular interactions. J Cell Biol. 1991;113(3):485-95.

23. Strelkov SV, et al. Divide-and-conquer crystallographic approach towards an atomic structure of intermediate filaments. J Mol Biol. 2001;306(4):773-81.

24. Ahn J, et al. Structural basis for lamin assembly at the molecular level. Nat Commun. 2019;10(1):3757.

25. Lee $\mathrm{CH}$, et al. Structure-function analyses of a keratin heterotypic complex identify specific keratin regions involved in intermediate filament assembly. Structure. 2020;28(3):355-362.e4

26. Herrmann H, Aebi U. Intermediate Filaments: Structure and Assembly. Cold Spring Harb Perspect Biol. 2016;8(11):a018242.

27. Parry DA, Marekov LN, Steinert PM. Subfilamentous protofibril structures in fibrous proteins: cross-linking evidence for protofibrils in intermediate filaments. J Biol Chem. 2001;276(42):39253-8.

28. Hol, E.M. and Y. Capetanaki, Type III Intermediate Filaments Desmin, Glial Fibrillary Acidic Protein (GFAP), Vimentin, and Peripherin. Cold Spring Harb Perspect Biol, 2017. 9(12).

29. Yuan A, et al. Neurofilaments and Neurofilament Proteins in Health and Disease. Cold Spring Harb Perspect Biol. 2017;9(4):a021642.

30. Tenga R, Medalia O. Structure and unique mechanical aspects of nuclear lamin filaments. Curr Opin Struct Biol. 2020;64:152-9.

31. Song $S$, et al. Functions of the intermediate filament cytoskeleton in the eye lens. J Clin Invest. 2009;119(7):1837-48.

32. Zimek A, Weber K. The organization of the keratin I and II gene clusters in placental mammals and marsupials show a striking similarity. Eur J Cell Biol. 2006;85(2):83-9.

33. Peter A, Stick R. Evolutionary aspects in intermediate filament proteins. Curr Opin Cell Biol. 2015;32:48-55.

34. Shaw PH, Held WA, Hastie ND. The gene family for major urinary proteins: expression in several secretory tissues of the mouse. Cell. 1983;32(3):755-61.

35. Ustariz CR, et al. Major HLA histocompatibility system as a possible genetic marker in haemophilia A and Von Willebrand's disease (author's transl). Sangre (Barc). 1978;23(3):314-9.

36. Jackson BC, et al. Update of the human secretoglobin (SCGB) gene superfamily and an example of "evolutionary bloom" of androgenbinding protein genes within the mouse Scgb gene superfamily. Hum Genom. 2011;5(6):691-702.

37. Nelson DR, et al. Comparison of cytochrome P450 (CYP) genes from the mouse and human genomes, including nomenclature recommendations for genes, pseudogenes and alternative-splice variants. Pharmacogenetics. 2004;14(1):1-18.

38. Feyereisen R. Origin and evolution of the CYP4G subfamily in insects, cytochrome P450 enzymes involved in cuticular hydrocarbon synthesis. Mol Phylogenet Evol. 2020;143:106695.

39. Perng MD, Zhang Q, Quinlan RA. Insights into the beaded filament of the eye lens. Exp Cell Res. 2007;313(10):2180-8.

40. Krushna Padhi B, Akimenko MA, Ekker M. Independent expansion of the keratin gene family in teleostean fish and mammals: an insight from phylogenetic analysis and radiation hybrid mapping of keratin genes in zebrafish. Gene. 2006;368:37-45.

41. Zimek A, Weber K. Terrestrial vertebrates have two keratin gene clusters; striking differences in teleost fish. Eur J Cell Biol. 2005;84(6):623-35.

42. Degl'Innocenti $A$, et al. A purely bioinformatic pipeline for the prediction of mammalian odorant receptor gene enhancers. BMC Bioinformatics. 2019;20(1):474.

43. Ribeiro DM, et al. The molecular basis, genetic control and pleiotropic effects of local gene co-expression. Nat Commun. 2021;12(1):4842.

44. Bunick CG, Milstone LM. The X-ray crystal structure of the keratin 1-Keratin 10 Helix 2B heterodimer reveals molecular surface properties and biochemical insights into human skin disease. J Invest Dermatol. 2017;137(1):142-50.
45. Ehrlich F, et al. Comparative genomics suggests loss of keratin K24 in three evolutionary lineages of mammals. Sci Rep. 2019;9(1):10924.

46. Strnad $P$, et al. Unique amino acid signatures that are evolutionarily conserved distinguish simple-type, epidermal and hair keratins. J Cell Sci. 2011;124(Pt 24):4221-32.

47. Kimura Y, Nikaido M. Conserved keratin gene clusters in ancient fish: An evolutionary seed for terrestrial adaptation. Genomics. 2021;113(1 Pt 2):1120-8.

48. Pekny M, Lane EB. Intermediate filaments and stress. Exp Cell Res. 2007;313(10):2244-54.

49. Vandebergh W, Bossuyt F. Radiation and functional diversification of alpha keratins during early vertebrate evolution. Mol Biol Evol. 2012;29(3):995-1004.

50. Alibardi $\mathrm{L}$. The process of cornification evolved from the initial keratinization in the epidermis and epidermal derivatives of vertebrates: a new synthesis and the case of sauropsids. Int Rev Cell Mol Biol. 2016;327:263-319.

51. Ehrlich F, et al. Differential evolution of the epidermal keratin cytoskeleton in terrestrial and aquatic mammals. Mol Biol Evol. 2019;36(2):328-40.

52. Albalat R, Canestro C. Evolution by gene loss. Nat Rev Genet. 2016;17(7):379-91.

53. Consortium GT. The Genotype-Tissue Expression (GTEx) project. Nat Genet. 2013;45(6):580-5.

54. Moll R, et al. The catalog of human cytokeratins: patterns of expression in normal epithelia, tumors and cultured cells. Cell. 1982;31(1):11-24.

55. Fuchs $\mathrm{E}$, Green $\mathrm{H}$. Changes in keratin gene expression during terminal differentiation of the keratinocyte. Cell. 1980;19(4):1033-42.

56. Kim J, Villadsen R. The expression pattern of epidermal differentiation marker Keratin 10 in the normal human breast and breast cancer cells. J Histochem Cytochem. 2020;68(8):561-70.

57. Adly MA, Hussein MR. Expression of cytokeratin 10 protein in the human testis showing normal and abnormal spermatogenesis. Ultrastruct Pathol. 2011;35(5):209-13.

58. Carrilho C, et al. Keratins $8,10,13$, and 17 are useful markers in the diagnosis of human cervix carcinomas. Hum Pathol. 2004;35(5):546-51.

59. Soultanova A, et al. Terminally differentiated epithelial cells of the thymic medulla and skin express nicotinic acetylcholine receptor subunit alpha 3. Biomed Res Int. 2014;2014:757502.

60. Cunha GR, et al. Molecular mechanisms of development of the human fetal female reproductive tract. Differentiation. 2017;97:54-72.

61. Zaina $S$, et al. Local reduction of organ size in transgenic mice expressing a soluble insulin-like growth factor II/mannose-6-phosphate receptor. Endocrinology. 1998;139(9):3886-95.

62. Roth $\mathrm{W}$, et al. Keratin 1 maintains skin integrity and participates in an inflammatory network in skin through interleukin-18. J Cell Sci. 2012;125(Pt 22):5269-79.

63. Dong $X$, et al. Critical role of Keratin 1 in maintaining epithelial barrier and correlation of its down-regulation with the progression of inflammatory bowel disease. Gene. 2017;608:13-9.

64. Chen W, et al. Silencing of keratin 1 inactivates the Notch signaling pathway to inhibit renal interstitial fibrosis and glomerular sclerosis in uremia. J Cell Physiol. 2020;235(2):1674-88.

65. Schaller G, Genz T. Immunohistochemical detection of keratins 1 and 13 as differentiation markers in the hormone-dependent human vaginal epithelium. Gynecol Obstet Invest. 1990;30(2):94-6.

66. Zhou XM, et al. The complete sequence of the human intermediate filament chain keratin 10. Subdomainal divisions and model for folding of end domain sequences. J Biol Chem. 1988;263(30):15584-9.

67. Toivola DM, et al. Cellular integrity plus: organelle-related and protein-targeting functions of intermediate filaments. Trends Cell Biol. 2005;15(11):608-17.

68. Casanova ML, et al. Exocrine pancreatic disorders in transsgenic mice expressing human keratin 8. J Clin Invest. 1999;103(11):1587-95.

69. Kuruc N, Franke WW. Transient coexpression of desmin and cytokeratins 8 and 18 in developing myocardial cells of some vertebrate species. Differentiation. 1988;38(3):177-93.

70. Bocker W, et al. Common adult stem cells in the human breast give rise to glandular and myoepithelial cell lineages: a new cell biological concept. Lab Invest. 2002;82(6):737-46. 
71. Polari L, et al. Keratin intermediate filaments in the colon: guardians of epithelial homeostasis. Int J Biochem Cell Biol. 2020;129:105878.

72. Gava N, et al. Global gene expression profiles of ovarian surface epithelial cells in vivo. J Mol Endocrinol. 2008;40(6):281-96.

73. Gires $\mathrm{O}$, et al. Cytokeratin 8 associates with the external leaflet of plasma membranes in tumour cells. Biochem Biophys Res Commun. 2005;328(4):1154-62.

74. Salas PJ, Forteza R, Mashukova A. Multiple roles for keratin intermediate filaments in the regulation of epithelial barrier function and apico-basal polarity. Tissue Barriers. 2016;4(3):e1178368.

75. Abe M, Oshima RG. A single human keratin 18 gene is expressed in diverse epithelial cells of transgenic mice. J Cell Biol. 1990;111(3):1197-206.

76. Jahn $\mathrm{L}$, et al. Cytokeratins in certain endothelial and smooth muscle cells of two taxonomically distant vertebrate species Xenopus laevis and man. Differentiation. 1987:36(3):234-54.

77. Drew JE, et al. Predictive gene signatures: molecular markers distinguishing colon adenomatous polyp and carcinoma. PLoS ONE. 2014;9(11):e113071.

78. Hasegawa T, et al. Keratinocyte progenitor cells reside in human subcutaneous adipose tissue. PLoS ONE. 2015;10(2):e0118402.

79. Nickerson DW, et al. Quantification of cytokeratin 5 mRNA expression in the circulation of healthy human subjects and after lung transplantation. PLoS ONE. 2009:4(6):e5925.

80. Reis-Filho JS, et al. Distribution of p63, cytokeratins 5/6 and cytokeratin 14 in 51 normal and 400 neoplastic human tissue samples using TARP-4 multi-tumor tissue microarray. Virchows Arch. 2003;443(2):122-32.

81. Hoogduin $\mathrm{KJ}$, et al. BCL2 and keratin 5 define the uterine-cervixisthmus junction, a transition between endocervical and tubal-like epithelium. Int J Gynecol Pathol. 2013;32(1):122-30.

82. Salahudeen AA, et al. Progenitor identification and SARS-CoV-2 infection in human distal lung organoids. Nature. 2020;588(7839):670-5.

83. Amaro A, et al. Validation of proposed prostate cancer biomarkers with gene expression data: a long road to travel. Cancer Metastasis Rev. 2014;33(2-3):657-71.

84. Demirkesen C, Hoede N, Moll R. Epithelial markers and differentiation in adnexal neoplasms of the skin: an immunohistochemical study including individual cytokeratins. J Cutan Pathol. 1995;22(6):518-35.

85. Smedts F, et al. Expression of keratins 1, 6, 15, 16, and 20 in normal cervical epithelium, squamous metaplasia, cervical intraepithelial neoplasia, and cervical carcinoma. Am J Pathol. 1993;142(2):403-12.

86. Swensson O, et al. Specialized keratin expression pattern in human ridged skin as an adaptation to high physical stress. Br J Dermatol. 1998;139(5):767-75

87. Petzoldt J L, et al. Culture and characterisation of human urothelium in vivo and in vitro. Urol Res. 1994;22(2):67-74.

88. Yang $Y$, et al. Differential expression of cytokeratin mRNA and protein in normal prostate, prostatic intraepithelial neoplasia, and invasive carcinoma. Am J Pathol. 1997;150(2):693-704.

89. Troyanovsky SM, et al. Patterns of expression of keratin 17 in human epithelia: dependency on cell position. J Cell Sci. 1989;93(Pt 3):419-26.

90. Liu J, et al. Keratin 17 promotes lung adenocarcinoma progression by enhancing cell proliferation and invasion. Med Sci Monit. 2018;24:4782-90

91. Wang YF, et al. Overexpression of keratin 17 is associated with poor prognosis in epithelial ovarian cancer. Tumour Biol. 2013;34(3):1685-9.

92. Escobar-Hoyos LF, et al. Keratin 17 in premalignant and malignant squamous lesions of the cervix: proteomic discovery and immunohistochemical validation as a diagnostic and prognostic biomarker. Mod Pathol. 2014;27(4):621-30.

93. Kim HS, et al. Overexpression of cytokeratin 17 is associated with the development of papillary thyroid carcinoma and the presence of lymph node metastasis. Int J Clin Exp Pathol. 2015;8(5):5695-701.

94. Sanchez-Carbayo M, et al. Molecular profiling of bladder cancer using cDNA microarrays: defining histogenesis and biological phenotypes. Cancer Res. 2002:62(23):6973-80.

95. Ricciardelli $\mathrm{C}$, et al. Keratin 5 overexpression is associated with serous ovarian cancer recurrence and chemotherapy resistance. Oncotarget. 2017;8(11):17819-32.

96. Nieuwenhuis TO, et al. Consistent RNA sequencing contamination in GTEx and other data sets. Nat Commun. 2020;11(1):1933.
97. Li R, et al. Association of keratin 8/18 variants with non-alcoholic fatty liver disease and insulin resistance in Chinese patients: a case-control study. World J Gastroenterol. 2017;23(22):4047-53.

98. Tsai $\mathrm{JH}$, et al. Aggressive non-alcoholic steatohepatitis following rapid weight loss and/or malnutrition. Mod Pathol. 2017;30(6):834-42.

99. Strnad $P$, et al. Keratin variants associate with progression of fibrosis during chronic hepatitis C infection. Hepatology. 2006;43(6):1354-63.

100. Mazzalupo S, et al. Role for keratins 6 and 17 during wound closure in embryonic mouse skin. Dev Dyn. 2003;226(2):356-65.

101. Kim S, Wong P, Coulombe PA. A keratin cytoskeletal protein regulates protein synthesis and epithelial cell growth. Nature. 2006:441(7091):362-5.

102. Steen $K$, et al. A role for keratins in supporting mitochondrial organization and function in skin keratinocytes. Mol Biol Cell. 2020;31(11):1103-11.

103. Lessard JC, et al. Keratin 16 regulates innate immunity in response to epidermal barrier breach. Proc Natl Acad Sci USA. 2013;110(48):19537-42.

104. Lessard JC, Coulombe PA. Keratin 16-null mice develop palmoplantar keratoderma, a hallmark feature of pachyonychia congenita and related disorders. J Invest Dermatol. 2012;132(5):1384-91.

105. Wu TT, Eldirany SA, Bunick CG, Teng JMC. Genotype-structurotypephenotype correlations in patients with pachyonychia congenita. J Invest Dermatol. 2021;141(12):2876-2884.e4. https://doi.org/10.1016/j. jid.2021.03.035

106. Liu F, et al. Overexpression of cell surface cytokeratin 8 in multidrugresistant MCF-7/MX cells enhances cell adhesion to the extracellular matrix. Neoplasia. 2008;10(11):1275-84.

107. Yu YT, et al. Surface vimentin is critical for the cell entry of SARS-CoV. J Biomed Sci. 2016;23:14.

108. Suprewicz $L$ et al. Extracellular vimentin as a target against SARS-CoV-2 host cell invasion. bioRxiv, 2021.

109. Afgan $\mathrm{E}$, et al. The Galaxy platform for accessible, reproducible and collaborative biomedical analyses: 2018 update. Nucleic Acids Res. 2018;46(W1):W537-44.

\section{Publisher's Note}

Springer Nature remains neutral with regard to jurisdictional claims in published maps and institutional affiliations.

Ready to submit your research? Choose BMC and benefit from

- fast, convenient online submission

- thorough peer review by experienced researchers in your field

- rapid publication on acceptance

- support for research data, including large and complex data types

- gold Open Access which fosters wider collaboration and increased citations

- maximum visibility for your research: over $100 \mathrm{M}$ website views per year

At BMC, research is always in progress.

Learn more biomedcentral.com/submissions 